\title{
Class-II dihydroorotate dehydrogenases from three phylogenetically distant fungi support anaerobic pyrimidine biosynthesis
}

\author{
Jonna Bouwknegt ${ }^{1} \mathbb{0}$, Charlotte C. Koster ${ }^{1}$, Aurin M. Vos ${ }^{2}$, Raúl A. Ortiz-Merino ${ }^{1}$, Mats Wassink ${ }^{1}$, \\ Marijke A. H. Luttik', Marcel van den Broek', Peter L. Hagedoorn ${ }^{1}$ and Jack T. Pronk ${ }^{* *}$
}

\begin{abstract}
Background: In most fungi, quinone-dependent Class-II dihydroorotate dehydrogenases (DHODs) are essential for pyrimidine biosynthesis. Coupling of these Class-II DHODHs to mitochondrial respiration makes their in vivo activity dependent on oxygen availability. Saccharomyces cerevisiae and closely related yeast species harbor a cytosolic Class-I DHOD (Ura1) that uses fumarate as electron acceptor and thereby enables anaerobic pyrimidine synthesis. Here, we investigate DHODs from three fungi (the Neocallimastigomycete Anaeromyces robustus and the yeasts Schizosaccharomyces japonicus and Dekkera bruxellensis) that can grow anaerobically but, based on genome analysis, only harbor a Class-II DHOD.
\end{abstract}

Results: Heterologous expression of putative Class-II DHOD-encoding genes from fungi capable of anaerobic, pyrimidine-prototrophic growth (Arura9, SjURA9, DbURA9) in an S. cerevisiae ura1 $\triangle$ strain supported aerobic as well as anaerobic pyrimidine prototrophy. A strain expressing DbURA9 showed delayed anaerobic growth without pyrimidine supplementation. Adapted faster growing DbURA9-expressing strains showed mutations in FUM1, which encodes fumarase. GFP-tagged SjUra9 and DbUra9 were localized to S. cerevisiae mitochondria, while ArUra9, whose sequence lacked a mitochondrial targeting sequence, was localized to the yeast cytosol. Experiments with cell extracts showed that ArUra9 used free FAD and FMN as electron acceptors. Expression of SjURA9 in S. cerevisiae reproducibly led to loss of respiratory competence and mitochondrial DNA. A cysteine residue (C265 in SjUra9) in the active sites of all three anaerobically active Ura9 orthologs was shown to be essential for anaerobic activity of SjUra9 but not of ArUra9.

Conclusions: Activity of fungal Class-II DHODs was long thought to be dependent on an active respiratory chain, which in most fungi requires the presence of oxygen. By heterologous expression experiments in S. cerevisiae, this study shows that phylogenetically distant fungi independently evolved Class-II dihydroorotate dehydrogenases that enable anaerobic pyrimidine biosynthesis. Further structure-function studies are required to understand the mechanistic basis for the anaerobic activity of Class-II DHODs and an observed loss of respiratory competence in S. cerevisiae strains expressing an anaerobically active DHOD from Sch. japonicus.

Keywords: Yeast, Neocallimastigomycota, Oxygen, Anaerobiosis, Oxygen requirements, Uracil

*Correspondence: j.t.pronk@tudelft.nl

1 Department of Biotechnology, Delft University of Technology, van der Maasweg 9, 2629 HZ Delft, The Netherlands

Full list of author information is available at the end of the article

\section{Background}

Oxidation of dihydroorotate to orotate, an essential reaction for pyrimidine biosynthesis in all domains of life, is catalyzed by the flavoprotein dihydroorotate dehydrogenase (DHOD) $[1,2]$. Depending on the type of DHOD, different electron acceptors are used for re-oxidation 
of the flavin cofactor. Soluble Class-I DHODs include homodimeric fumarate-dependent type I-A enzymes and heterotetrameric $\mathrm{NAD}^{+}$-dependent type I-B enzymes $[3,4]$, while membrane-bound Class-II DHODs are quinone-dependent and coupled to respiration [5]. Protein sequence identity between Class-I and Class-II DHODs is only approximately $20 \%$ [6-8].

Class I-A and Class-II DHODs contain a single FMN cofactor per subunit. In heterotetrameric Class I-B enzymes, two subunits also each contain an FMN cofactor, while the other two both contain an FAD cofactor and an [2Fe-2S] cluster [4]. Class-I DHODs contain an active-site cysteine residue involved in deprotonation of $\mathrm{C} 5$ of dihydroorotate, while Class-II enzymes have a serine in the same position acting as the catalytic base [9-13].

Most eukaryotes harbor a monomeric Class-II DHOD that donates electrons to the quinone pool of the mitochondrial respiratory chain $[8,14-16]$. Bacterial Class-II DHODs have an $\mathrm{N}$-terminal sequence that localizes them to the inside of the cytoplasmic membrane, whereas eukaryotic enzymes are targeted to the outside of the mitochondrial inner membrane [5]. This mitochondrial targeting also applies to fungal Class-II DHODs, which include the Ura9 orthologs of yeasts such as Lachancea kluyveri and Schizosaccharomyces pombe [17]. Since respiration in yeasts requires oxygen as electron acceptor, reliance on these respiration-coupled enzymes precludes pyrimidine-prototrophic anaerobic growth [8].

Class-I DHODs predominantly occur in gram-positive bacteria and Archaea [18] but are also found in a small number of yeasts, including Saccharomyces cerevisiae and closely related species $[19,20]$. S. cerevisiae is among the few yeast species that are able to grow under strictly anaerobic conditions [21]. ScUra1, a Class-IA, fumarate-coupled DHOD, enables $S$. cerevisiae to synthesize pyrimidines in the absence of oxygen $[19,20]$. A small number of other Saccharomycetes, including Kluyveromyces lactis and L. kluyveri, harbor Ura1 as well as Ura9 orthologs [19, 22, 23]. Based on sequence similarity of yeast ScURA1 orthologs with Lactococcus genes, they are assumed to have been acquired by horizontal gene transfer $[15,19]$.

In line with a proposed essentiality of ScURA1 orthologs for anaerobic pyrimidine synthesis by yeasts [19, 24], replacement of ScURA1 by a Class-II DHOD gene from L. kluyveri (LkURA9) or Sch. pombe (SpURA3) yielded strains that were only pyrimidine prototrophic under aerobic conditions $[8,19]$. Conversely, replacement of ScURA1 by $L k U R A 1$ supported aerobic as well as anaerobic pyrimidine prototrophy [19]. Introduction of ScURA1 in URA9dependent yeasts was proposed as a metabolic engineering strategy for enabling anaerobic, pyrimidine-prototrophic growth of yeasts lacking a native ScURA1 ortholog [25].

The long-held assumption that expression of a Class-I DHOD is required for anaerobic pyrimidine biosynthesis in eukaryotes was first challenged when Dekkera bruxellensis, which only harbors a putative URA9 ortholog, was shown to grow anaerobically in pyrimidine-free media [26, 27]. A hypothesis that DbUra9 is able to use a non-quinone electron acceptor $[17,28,29]$ was, however, not experimentally tested.

We recently observed that $D$. bruxellensis may not be the only eukaryote in which anaerobic pyrimidine synthesis involves a Class-II DHOD. Inspection of the genome of the fission yeast Sch. japonicus, which shows fast anaerobic growth in synthetic media without uracil [30, 31], suggested that it only contains a URA9 ortholog. Moreover, genomes of Neocallimastigomycetes, a group of deepbranching, obligately anaerobic fungi that lack mitochondria and instead harbor hydrogenosomes [32, 33], also appeared to lack orthologs of soluble Class-I DHOD.

The goals of the present study were to investigate whether URA9 orthologs in eukaryotes capable of anaerobic growth indeed support anaerobic pyrimidine biosynthesis, and to gain more insight into underlying mechanisms and tradeoffs. To this end, we expressed putative Class-II DHOD genes from the obligately anaerobic Neocallimastigomycete Anaeromyces robustus (Arura9), the facultative anaerobes Sch. japonicus (SjURA9) and D. bruxellensis (DbURA9), as well as from the oxygen-requiring yeasts Ogataea parapolymorpha (OpURA9) and Kluyveromyces marxianus (KmURA9), in an S. cerevisiae ura1 1 background. After studying aerobic and anaerobic growth of the resulting strains in uracil-supplemented and uracil-free synthetic media, we analyzed subcellular localization of Ura9-eGFP fusion proteins in S. cerevisiae and assessed the impact of a conserved amino-acid substitution in anaerobically functional Ura9 orthologs. To identify possible natural electron acceptors, we performed enzyme assays in cell extracts of an S. cerevisiae strain expressing ArUra9 and resequenced the genomes of laboratory-evolved $S$. cerevisiae strains whose anaerobic growth depended on expression of DbURA9. We found that instead of quinone, ArUra9 uses free flavins as electron acceptors and that expression of SjURA9 in S. cerevisiae results in loss of respiration.

\section{Results}

Obligately anaerobic Neocallimastigomycetes and facultatively anaerobic yeasts harbor putative Class-II DHODs

A preliminary exploration of a set of selected fungal proteomes for Class-I and Class-II DHODs was based on a sequence similarity search with Class-I and Class-II 
enzymes of L. kluyveri (LkUra1 and LkUra9, respectively; [19]; Table 1) as queries. Consistent with earlier studies, the $S$. cerevisiae proteome showed a single sequence with strong similarity to LkUra1 (ScUra1; [10, 19, 23]), while proteomes of the facultatively fermentative yeasts $O$. parapolymorpha and $K$. marxianus, which both require oxygen for biosynthesis-related reactions [34, 35], yielded previously described Class-II DHOD sequences with strong homology to LkUra9 (OpUra9 and KmUra9, respectively; $[23,36])$. K. marxianus additionally showed a sequence with high sequence similarity to LkUra1 (KmUra1; [22]). The search also confirmed earlier reports that the facultatively anaerobic yeast $D$. bruxellensis only harbors a putative DHOD sequence with high similarity to LkUra9 (DbUra9; [17, 23, 28]).

Sequence comparison with LkUra1 provided no evidence for presence of a Class-I DHOD in the Neocallimastigomycetes Piromyces finnis, Neocallimastix californiae and $A$. robustus. Instead, these obligately anaerobic fungi yielded predicted protein sequences with high similarity to the Class-II DHOD LkUra9 (Table 1), which we tentatively called PfUra9, NcUra9 and ArUra9, respectively. A similar result was obtained for the facultatively anaerobic fission yeast Sch. japonicus [30, 31], whose putative Class-II DHOD was tentatively named SjUra9 (Table 1). Dependence of anaerobic, pyrimidineprototrophic growth of phylogenetically distant fungi on Class-II ('Ura9') DHODs would be remarkable in view of the well-documented coupling of canonical eukaryotic Class-II DHODs to aerobic mitochondrial respiration [5, $8,19,37,38]$.

To study the phylogeny of fungal Ura9 orthologs, the amino-acid sequence of LkUra9 was used as query for a sequence analysis with a hidden Markov model method
(HMMER; [39]) against all fungal proteomes in Uniprot [40], from which the best hits were used for orthology prediction (see "Methods"). A similar strategy was performed to obtain bacterial LkUra9 orthologs. A phylogenetic tree constructed based on the resulting 331 fungal and 73 bacterial Ura9 orthologs showed a clear separation of bacterial and fungal Ura9 orthologs (Fig. 1; Additional file 1, Additional file 2, Additional file 3). The topology of the tree followed fungal phylogeny, as exemplified by the LkUra9 ortholog of Sch. japonicus, which clustered with that of closely related species $S c h$. pombe whose Class-II DHOD was previously shown not to support anaerobic uracil prototrophy [8]. Similarly, the $D$. bruxellensis Ura9 ortholog clustered with those of other Pichiacaea yeasts, including O. parapolymorpha whose Ura9 ortholog does not support anaerobic uracil prototrophy (see below). These results indicated that, if Ura9 orthologs of Neocallimastigomycetes, D. bruxellensis and Sch. japonicus share properties that enable anaerobic pyrimidine synthesis, these are likely to have evolved independently, without involvement of horizontal gene transfer (HGT). Evolution of oxygen-independent pyrimidine synthesis in these fungi would then differ from the proposed HGT-mediated acquisition of a respirationindependent Class-I DHOD by an ancestor of S. cerevisiae $[19,20]$ and from proposed HGT-driven adaptations of Sch. japonicus [31, 41] and Neocallimastigomycetes $[42,43]$ to bypass other oxygen requirements for biosynthetic processes.

\section{Heterologous URA9 genes complement aerobic pyrimidine auxotrophy of ura $1 \Delta$ S. cerevisiae}

To assess functionality of the putative Class-II DHOD genes of the Neocallimastigomycete A. robustus and

Table 1 L. kluyveri LkUra1 and LkUra9 sequence homology results using selected fungal proteomes

\begin{tabular}{|c|c|c|c|c|c|c|c|c|c|}
\hline \multirow[t]{2}{*}{ Subject proteome } & \multirow{2}{*}{$\begin{array}{l}\text { Resulting } \\
\text { GenBank } \\
\text { accession }\end{array}$} & \multicolumn{2}{|c|}{ Query coverage (\%) } & \multicolumn{2}{|l|}{ E-value } & \multicolumn{2}{|c|}{ Identity (\%) } & \multicolumn{2}{|c|}{ Interpretation } \\
\hline & & LkUra1 & LkUra9 & LkUra1 & LkUra9 & LkUra1 & LkUra9 & Ura1 & Ura9 \\
\hline D. bruxellensis [91] & XP_041139490.1 & 95 & 90 & $5 \cdot 10^{-17}$ & $4 \cdot 10^{-145}$ & 24.5 & 50.9 & No & Yes \\
\hline A. robustus $[108,109]$ & ORX87218.1 & 56 & 77 & $3 \cdot 10^{-9}$ & $2 \cdot 10^{-85}$ & 27.0 & 44.6 & No & Yes \\
\hline P. finnis $[108,109]$ & ORX52621.1 & 56 & 77 & $5 \cdot 10^{-9}$ & $7 \cdot 10^{-86}$ & 24.9 & 44.9 & No & Yes \\
\hline N. californiae [108] & ORY72481.1 & 54 & 74 & $2 \cdot 10^{-8}$ & $5 \cdot 10^{-82}$ & 27.7 & 44.9 & No & Yes \\
\hline Sch.japonicus [110] & XP_002171492.1 & 63 & 97 & $2 \cdot 10^{-13}$ & $1 \cdot 10^{-113}$ & 30.6 & 44.3 & No & Yes \\
\hline S. cerevisiae [111] & NP_012706.1 & 100 & 71 & 0.0 & $4 \cdot 10^{-9}$ & 80.3 & 23.8 & Yes & No \\
\hline \multirow[t]{2}{*}{ K. marxianus [112] } & XP_022674337.1 & 100 & 73 & 0.0 & $2 \cdot 10^{-12}$ & 77.1 & 25.1 & Yes & No \\
\hline & XP_022675611.1 & 94 & 95 & $6 \cdot 10^{-11}$ & 0.0 & 24.0 & 73.7 & No & Yes \\
\hline O. parapolymorpha [113] & XP_013936870.1 & 95 & 91 & $1 \cdot 10^{-9}$ & 0.0 & 23.0 & 63.4 & No & Yes \\
\hline
\end{tabular}

Proteomes of the Neocallimastigomycetes A. robustus (NCBI taxid 1754192), P. finnis (1754191), N. californiae (1754190), and the yeasts Sch. japonicus (402676), D. bruxellensis (5007), K. marxianus (1003335), O. parapolymorpha (871575) and S. cerevisiae (559292) were subjected to blastp searches using LkUra1 (DHOD Class I-A, UniProt KB accession number Q7Z892) and LkUra9 (DHOD Class II, accession number Q6V3W9) amino acid sequences as queries 


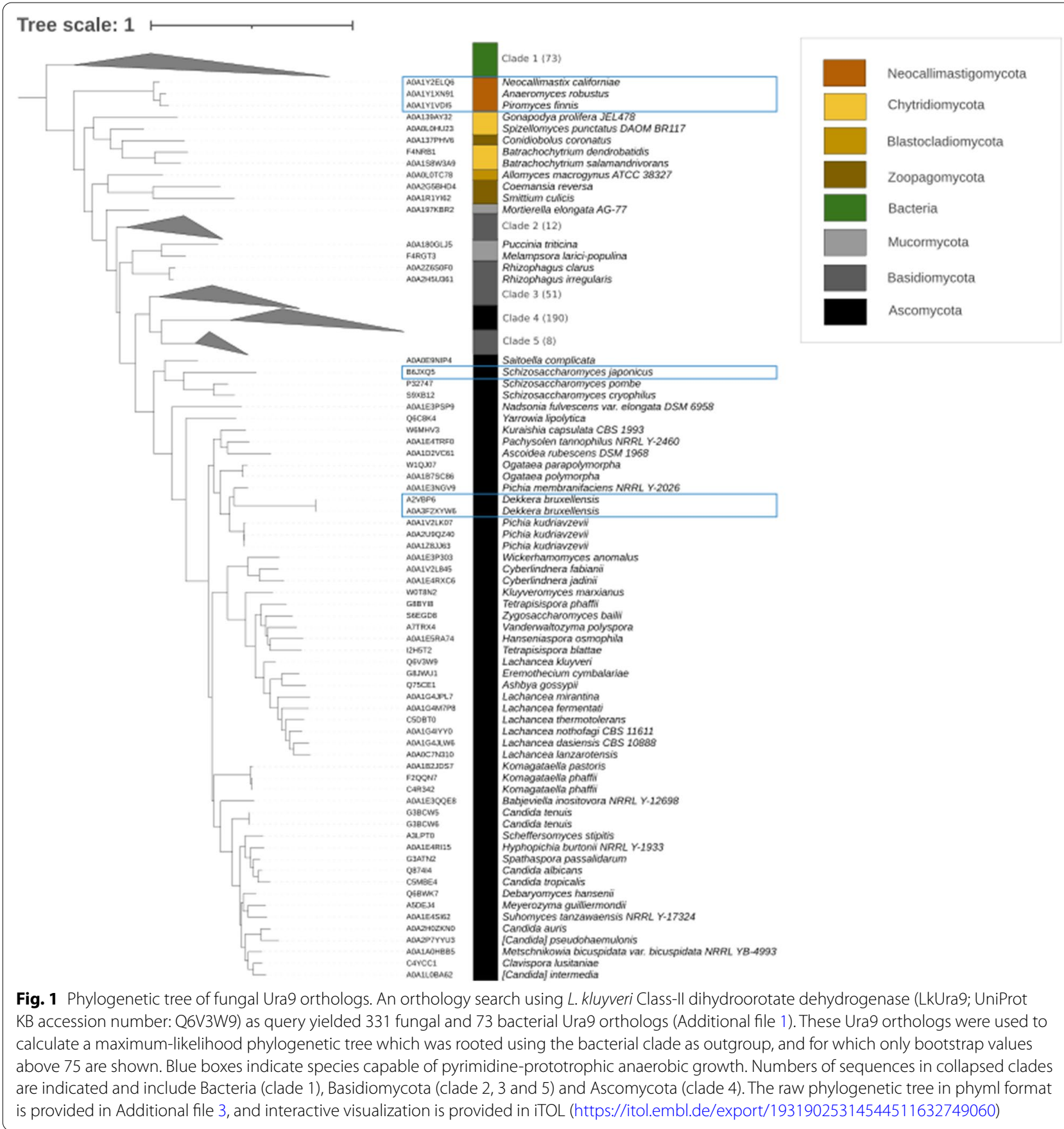

of the facultatively anaerobic yeasts $S c h$.japonicus and D. bruxellensis, the ScURA1 open-reading frame of S. cerevisiae was replaced by expression cassettes for codon-optimized Arura9, SjURA9 or DbURA9 coding sequences (Additional file 4). In addition, strains were constructed in which ScURA1 was replaced by expression cassettes for URA9 orthologs of the aerobic yeasts $K$. marxianus and O. parapolymorpha.

The $S$. cerevisiae reference strain CEN.PK113-7D (URA1) grew fast $\left(0.36 \mathrm{~h}^{-1}\right)$ in anaerobic cultures on glucose-containing synthetic media with and without uracil (SMUD + ura and SMUD, respectively), 
while the congenic ura1 $\Delta$ strain IMK824 only grew on SMUD + ura $\left(0.30 \mathrm{~h}^{-1}\right.$, Fig. 2, Additional file 5: Table S1). The lower specific growth rate of the uracilauxotrophic strain IMK824 on SMUD + ura probably reflected a growth-limiting rate of uracil uptake [44]. $S$. cerevisiae ura1 $1 \Delta$ strains carrying expression cassettes for Arura9, DbURA9, KmURA9 or OpURA9 all grew aerobically on SMUD + ura and SMUD, at specific growth rates of $0.34 \mathrm{~h}^{-1}$ to $0.35 \mathrm{~h}^{-1}$. A ura1 $1 \Delta$ strain expressing SjURA9 grew almost two-fold slower $\left(0.17-0.19 \mathrm{~h}^{-1}\right)$ on SMUD than the other uracil-prototrophic strains (Fig. 2, Additional file 5: Table S1). The growth rate of the SjURA9-expressing strain on SMUD + ura also was approximately one-third lower than that of the ura1 $\triangle$ strain IMK824, indicating that expression of SjURA9 negatively affected aerobic growth of $S$. cerevisiae on glucose.

These results showed that ArUra9, SjUra9 and DbUra9 are functional DHODs that complement a ura1 1 mutation in S. cerevisiae under aerobic conditions. A similar complementation of S. cerevisiae ura1 null mutants was previously demonstrated for Class-II DHODs of the oxygen-requiring yeasts L. kluyveri (LkUra9 [19]) and Sch. pombe (SpUra3 [8]).
Class II-DHODs of A. robustus, D. bruxellensis and Sch. japonicus support anaerobic growth of an S. cerevisiae ura $1 \Delta$ strain

A. robustus, D. bruxellensis and Sch. japonicus were previously reported to grow anaerobically in synthetic media without pyrimidine supplementation [26, 27, 30, 45]. While, based on genome-sequence analysis, previous studies $[17,23,28,29]$ inferred that anaerobic pyrimidine prototrophy of $D$. bruxellensis was supported by a ClassII DHOD (DbUra9), its activity was not experimentally confirmed. To assess anaerobic functionality of ArUra9, DbUra9 and SjUra9, we investigated whether these ClassII DHODs could support anaerobic, pyrimidine-prototrophic growth of ura $1 \Delta S$. cerevisiae strains.

As anticipated, the reference strain S. cerevisiae CEN. PK113-7D, which expresses the native fumarate-dependent Class I-A DHOD ScUra1, showed similar specific growth rates in anaerobic cultures on SMUD and SMUD + ura (0.24-0.25 $\mathrm{h}^{-1}$, Fig. 2, Additional file 5: Table S1), while strain IMK824 (ura1 $\Delta$ ) only grew anaerobically $\left(0.26 \mathrm{~h}^{-1}\right)$ on SMUD + ura (Fig. 2, Additional file 5: Table S1). Strains IMI446 and IMI447, in which ScURA1 was replaced by URA9 genes of the aerobic yeasts $K$. marxianus and O. parapolymorpha, only grew

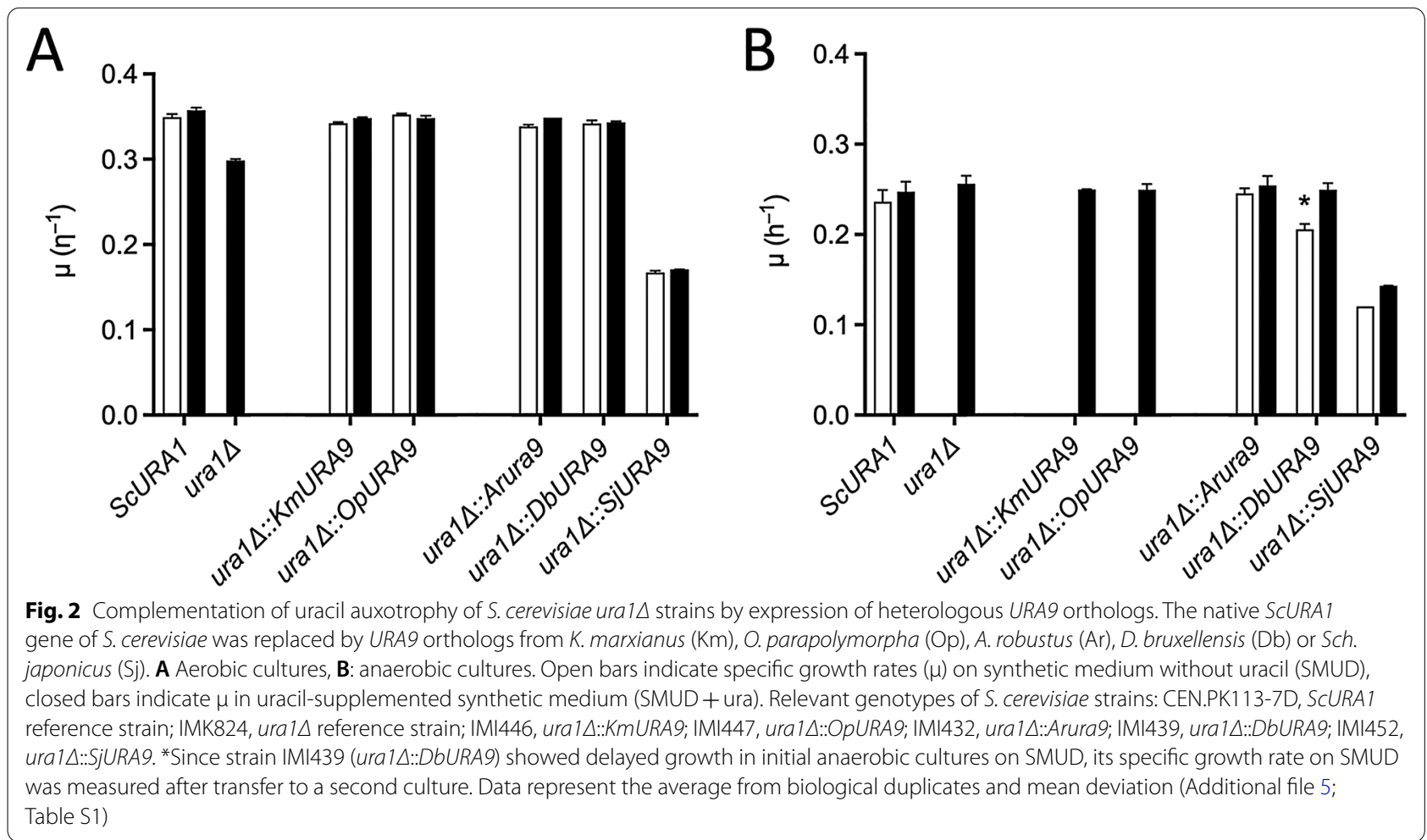


anaerobically when media were supplemented with uracil (Fig. 2, Additional file 5: Table S1). These results are in line with the coupling of canonical eukaryotic Class-II DHODs to the quinone pool of the mitochondrial respiratory chain $[5,8,19,37,38]$ and with previous studies by Gojković et al. [19] and Nagy et al. [8].

In contrast to expression of URA9 orthologs from oxygen-requiring yeasts, expression of Arura9 in S. cerevisiae supported fast anaerobic growth $\left(0.25 \mathrm{~h}^{-1}\right)$ on SMUD (Fig. 2, Additional file 5: Table S1). An S. cerevisiae strain in which ScURA1 was replaced by a SjURA9 expression cassette also grew anaerobically on SMUD as well as SMUD + ura, at an approximately two-fold lower growth rate than that of the strain expressing Arura9 (Fig. 2, Additional file 5: Table S1).

Strain IMI439, in which DbURA9 replaced ScURA1, did not show anaerobic growth on SMUD during the first $30 \mathrm{~h}$ of incubation (Additional file 5: Figure S1). When, after $68 \mathrm{~h}, \mathrm{OD}_{600}$ had increased to 2.9 , the strain was transferred to fresh SMUD, resulting in immediate anaerobic growth. In a parallel experiment with strain IMI447, which expressed KmURA9, no growth was observed upon transfer to fresh SMUD.

These results indicated that Class-II DHODs from the Neocallimastigomycete $A$. robustus and from the facultatively anaerobic yeast Sch. japonicus support pyrimidine synthesis under anaerobic conditions. The delayed anaerobic growth of a ura1 $1 \Delta$ strain expressing DbURA9 suggested that anaerobic functionality of DbURA9 in $S$. cerevisiae requires physiological or genetic adaptations.

\section{Fast anaerobic, uracil-prototrophic growth} of DbUra9-dependent S. cerevisiae strains correlates with mutations in FUM1

To further explore anaerobic functionality of DbUra9, we investigated whether adaptation of $S$. cerevisiae IMI439 (ura1 $\triangle:$ :DbURA9) to anaerobic, pyrimidineprototrophic growth (Additional file 5; Figure S1) was associated with mutations in the DbURA9 expression cassette or in native $S$. cerevisiae genes. Two independent anaerobic cultures of this strain on SMUD were incubated until growth occurred and then transferred to fresh anaerobic SMUD, in which they instantaneously grew (Additional file 5: Figure S1). Upon reaching stationary phase, two single colonies were isolated from each culture. To check whether these isolates (IMS1167, IMS1168, IMS1169 and IMS1170) had acquired stable mutations that stimulated anaerobic, uracil-prototrophic growth, they were first grown under non-selective conditions (aerobic growth on SMUD + ura) and then transferred to anaerobic medium with a reduced uracil content (SMUD + ura0.1). Inoculation of anaerobic cultures on SMUD with the resulting anaerobic, pyrimidine-limited cultures resulted in instantaneous anaerobic growth of all four isolates (Additional file 5: Table S1). Comparison of whole-genome sequences of the four isolates with that of their parental strain IMI439 (ura1 $\triangle:: D b U R A 9)$ revealed no mutations in the DbURA 9 expression cassette. Strains IMS1167 and IMS1168, which originated from the same anaerobically adapted culture, both contained point mutations in VPS1 $\left(V P S 1^{1410 \mathrm{~L}}\right)$, which encodes a dynamin-like GTPase involved in vacuolar sorting and in FUM1 $\left(F U M 1^{\mathrm{M} 432 \mathrm{I}}\right.$ ), encoding fumarase (Table 2). Strains IMS1169 and IMS1170, which were isolated from the second anaerobically adapted culture, each harbored a different mutation in FUM1 (FUM1 ${ }^{A 294 V}$ and $F U M 1^{\text {T218M }}$, respectively; Table 2). These results indicated that anaerobic activity of DbUra9 in S. cerevisiae does not require changes in its protein sequence but, based on the presence of mutations in FUM1 in all four isolates, may depend on the intracellular fumarate concentration.

\section{A cysteine residue in the active site of $\mathrm{SjUra9}$ is required for activity under anaerobic conditions}

To identify potentially relevant differences in the aminoacid sequences of Ura9 orthologs from oxygen-requiring yeast strains and those of anaerobically functioning Ura9 enzymes, sequences of ArUra9, SjUra9, DbUra9, KmUra9 and OpUra9 were subjected to a multiple sequence alignment, along with those of the characterized Class II DHODs of L. kluyveri (LkUra9l), Sch. pombe (SpUra3), and DHOD sequences of the Neocallimastigomycetes $N$. californiae (NcUra9) and P. finnis (PfUra9) (Additional file 5: Figure S2). In comparison with the yeast Ura9 sequences, those of the three Neocallimastigomycetes showed a 76-81 amino-acid truncation $\mathrm{N}$-terminal truncation. In canonical fungal Ura9 enzymes, the N-terminus contains a mitochondrial targeting sequence $[5,38]$ and is proposed to be involved in quinone binding [46, 47].

Table 2 Mutations in DbURA9 expressing strains, evolved for anaerobic pyrimidine prototrophy

\begin{tabular}{llll}
\hline Evolution line & Strain & Mutations & \\
\cline { 3 - 4 } & & Vps1 & Fum1 \\
\hline Flask 1 & IMS1167 & I410L & M432I \\
Flask 2 & IMS1168 & I410L & M432I \\
& IMS1169 & - & A294V \\
& IMS1170 & - & I218M \\
\hline
\end{tabular}

Two cultures of IMI439 (ura1A::DbURA9) were independently evolved under anaerobic conditions on SMUD (Additional file 5: Figure S1). Two single colony isolates from each flask were subjected to whole-genome resequencing and predicted amino-acid substitutions were only found in Vps1 and Fum1 
The Neocallimastigomycete Ura9 sequences as well as those of the two facultatively anaerobic yeasts (DbUra9 and $\mathrm{SjU}$ ra9) contained a cysteine residue instead of the conserved serine residue that acts as catalytic base in canonical Class-II DHODs [11, 12, 48]. Of 331 fungal Ura9 orthologs (Additional file 1) only three additional proteins (from Coemansia reversa, Smittium culisis and Gonapodya prolifera) harbored a cysteine at this position, but did not show an N-terminal truncation (Additional file 1 and Additional file 5: Figure S2).

Since soluble fumarate- and $\mathrm{NAD}^{+}$-dependent ClassI DHODs also use a cysteine residue as catalytic base $[12,13]$, we investigated the relevance this residue for in vivo activity of ArUra9 and SjUra9 in S. cerevisiae. Because of the slow adaptation to anaerobic, uracil-prototrophic growth of IMI439 (ura1D::DbURA9), we did not include DbUra9 in these experiments. Point mutations were introduced in Arura9 (C168S) and SjURA9 (C265S) to change the cysteine codon for a serine, yielding strains IMG007 (ura14::Arura9 ${ }^{\mathrm{C} 168 \mathrm{~S}}$ ) and IMG008 (ura1 $1:: S j U R A 9^{\mathrm{C} 265 \mathrm{~S}}$ ). In addition, a point mutation in the corresponding serine codon of KmURA9 was introduced to change it to a cysteine, yielding strain IMG005 (ura1A::KmURA9 ${ }^{\mathrm{S} 263 \mathrm{C}}$ ).

Changing the active-site serine residue (S263) of KmUra9 to a cysteine did not affect aerobic growth of $S$. cerevisiae, as shown by identical specific growth rates of strains IMG005 (ura1 $1:$ KmURA9 $9^{\text {S263C }}$ ) and IMI446 (ura1 $1:$ KmURA9) on SMUD (Fig. 3, Additional file 5: Table S1). This result indicated that, at least in KmUra9, the serine catalytic base that is strongly conserved in canonical Class-II DHODs is not essential for activity under aerobic conditions. However, strain IMG005 did not grow anaerobically without uracil supplementation (Fig. 3, Additional file 5: Table S1), indicating that replacement of the catalytic-base serine residue of KmUra9 by a cysteine is not sufficient to enable anaerobic functionality in $S$. cerevisiae.

Replacing the active-site cysteine residue in ArUra9 by a serine residue did not cause a different aerobic or anaerobic growth rate on SMUD of strain IMG007 (ura1 $1::$ Arura $^{\mathrm{C} 168 \mathrm{~S}}$ ) relative to its parental strain (Fig. 3, Additional file 5: Table S1). Apparently, despite our observation that an active-site cysteine residue occurred in different anaerobically active Ura9 orthologs, it is not required for anaerobic functionality of ArUra9 in S. cerevisiae. A strikingly different result was obtained upon changing the cysteine residue in the active site of SjUra9 to a serine. Under aerobic conditions, exponential growth of strain IMG008 (ura1 $1:: S j U R A 9^{\mathrm{C} 265 S}$ ) on SMUD was nearly two-fold slower than that of its parental strain IMI452 (ura1 $\triangle:: S j U R A 9$ ). In contrast, strain IMG008 (ura1 $\triangle:: S j U R A 9^{\mathrm{C} 265 \mathrm{~S}}$ ) failed to grow on SMUD

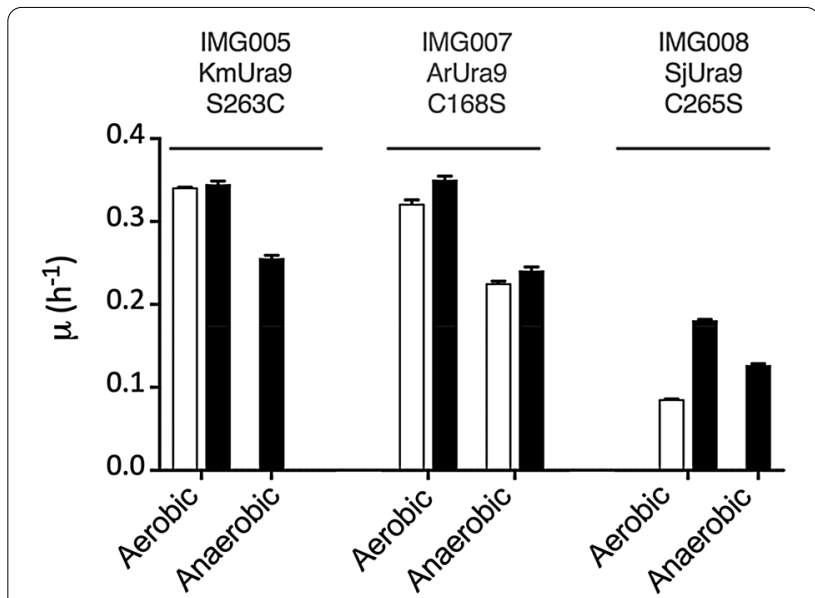

Fig. 3 Complementation of uracil auxotrophy of S. cerevisiae ura $1 \triangle$ strains by expressing mutated heterologous URA9 orthologs. The native SCURA1 gene of S. cerevisiae was replaced by URA9 orthologs from K. marxianus (Km), A. robustus (Ar) or Sch.japonicus (Sj) with single-nucleotide mutations affecting a specific serine or cysteine residue. Open bars indicate specific growth rates $(\mu)$ on synthetic medium without uracil (SMUD), closed bars indicate $\mu$ in uracil-supplemented synthetic medium (SMUD + ura). Cultures were grown aerobically or anaerobically as indicated in the Figure. Relevant genotypes of S. cerevisiae strains: IMG005; ura1 $\triangle:$ KMUURA9 $9^{5263 C}$, IMG007; ura1 $\triangle:$ Arura9 $^{\text {C168S }}$, IMG008; ura1 $: .: S j U R A 9^{C 265 S}$. Data represent the average from biological duplicates and mean deviation (Additional file 5: Table S1)

under anaerobic conditions. These results indicated that the active-site cysteine residue in SjUra9, but not in ArUra9, is required for DHOD activity under anaerobic conditions.

\section{Subcellular localization of heterologous Ura9 orthologs expressed in S. cerevisiae}

To investigate subcellular localization of Ura9 orthologs, eGFP fusions of anaerobically active ArUra9, DbUra9 and SjUra9, as well as of OpUra9, were expressed from multicopy (mc) plasmids in an S. cerevisiae ura $1 \Delta$ strain, followed by fluorescence-microscopy analysis of the resulting strains (Fig. 4). A co-expressed mRuby2 fluorescent protein fused to the preCOX4 mitochondrial targeting sequence [49] was used as marker for mitochondrial localization.

In S. cerevisiae strains IME600 (ura1D mcArura9eGFP), IME601 (ura1 $\mathrm{mcDbURA9-eGFP)} \mathrm{and} \mathrm{IME604}$ (ura1A mcOpURA9-eGFP), mRuby2 fluorescence showed multiple small mitochondria, a pattern that is representative for respiring cells [50]. Consistent with the localization of canonical eukaryotic Class-II DHODs [5], OpUra9-eGFP fluorescence overlapped with that of preCOX4-MTS-mRuby2 (Fig. 4A). A similar co-localization of DbUra9-eGFP and mRuby in 


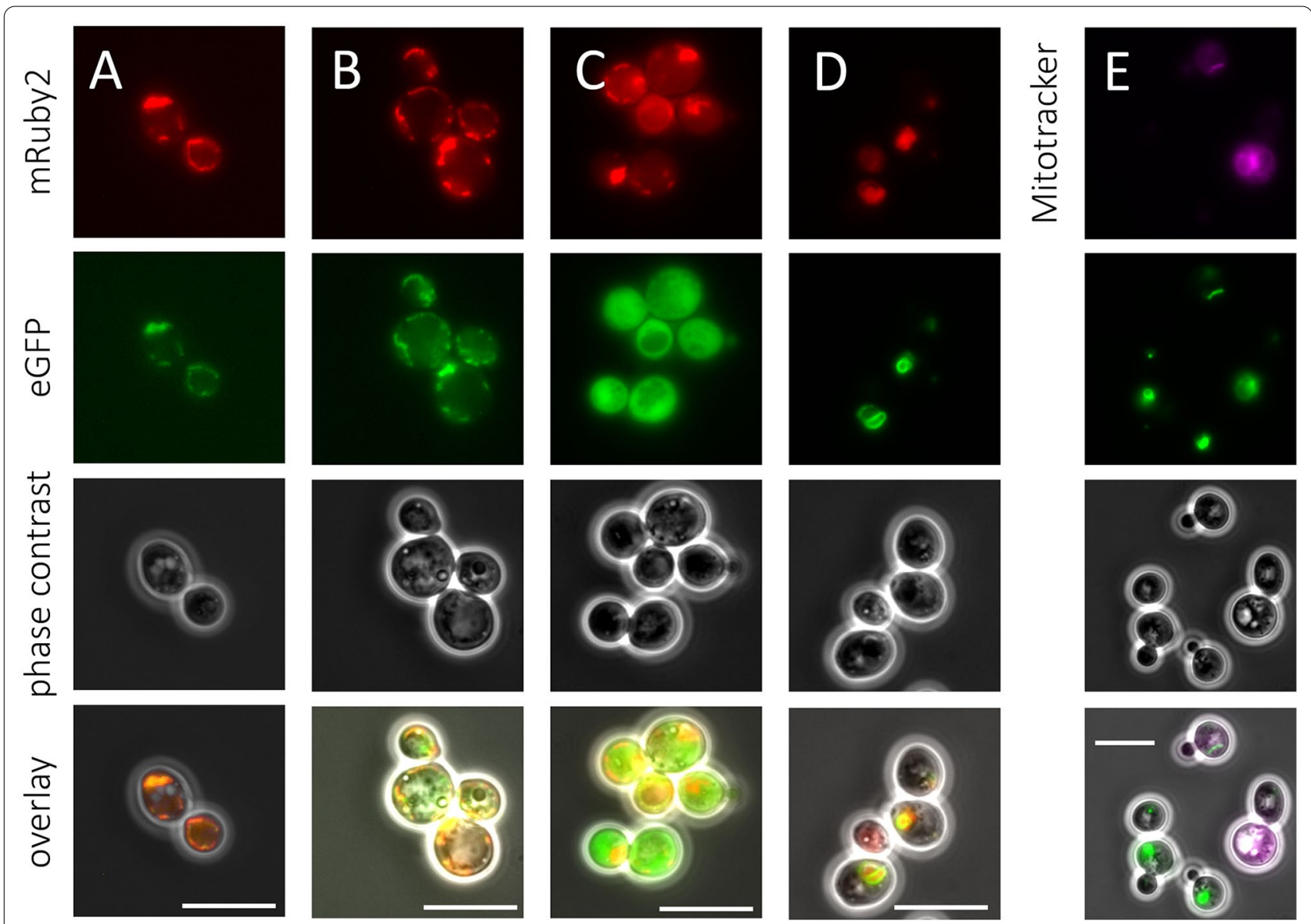

Fig. 4 Fluorescence microscopy images of S. cerevisiae strains expressing mitochondrially targeted mRuby2 together with different Ura9 orthologs fused to eGFP. Cells were grown on SMUD for at least two duplications and fluorescence of eGFP, mRuby and MitoTracker Deep Rep FM was detected by fluorescence microscopy. For each strain, from top to bottom: mRuby2/MitoTracker Deep Red FM fluorescence specifically localized to mitochondria, indicating localization of mitochondrial mass; eGFP fluorescence, tagged to different Ura9 orthologs, indicating subcellular Ura9 localization; phase-contrast image; and an overlay of all channels. From left to right; A IME604, expressing OpURA9-eGFP, B IME601 (DbURA9-eGFP), C IME600 (Arura9-eGFP), D IME602, (SjURA9-eGFP) and E IME602 stained with MitoTracker Deep Red FM. Scale bars are equivalent to $10 \mu \mathrm{m}$. Pictures are a representation of the full culture

strain IME601 indicated that, despite its activity under anaerobic conditions, DbUra9 was targeted to mitochondria (Fig. 4B). Consistent with the N-terminal truncation of Ura9 orthologs from Neocallimastigomycetes, but in striking contrast to canonical eukaryotic Class-II DHODs, ArUra9-eGFP was clearly localized to the yeast cytosol (Fig. 4C).

In strain IME602 (ura1A mcSjURA9-eGFP), mRuby2 fluorescence did not reveal the punctuate mitochondrial structures seen in the other strains. Instead, eGFP fluorescence was associated with tubular structures, that partially overlapped with a less defined mRuby 2 fluorescence (Fig. 4D). Although elongated mitochondrial morphologies occur in fermenting $S$. cerevisiae cells [50], the diffuse mRuby2 fluorescence in strain IME602 did not allow for clear localization of SjUra9eGFP. This strain was therefore also stained with the dye MitoTracker Deep Red. This approach only yielded vague tube-like structures or no fluorescence at all (Fig. 4E). Since staining by both preCOX4-MTSmRuby2 and MitoTracker Deep Red depend on mitochondrial membrane potential [49], we hypothesized that expression of SjURA9 reduces or abolishes mitochondrial membrane potential, possibly as a consequence of a loss of respiratory capacity [51].

\section{Expression of SjURA9 in S. cerevisiae causes loss} of respiratory capacity and mitochondrial DNA

To investigate whether expression of SjURA9 causes loss of respiratory capacity, SjURA9-expressing S. cerevisiae strains were tested for their ability to grow on non-fermentable carbon sources. In contrast to the reference strain S. cerevisiae CEN.PK113-7D (Fig. 5A), three independently constructed strains expressing SjURA9 
(IMI452, IMI462 and IME571; Additional file 5: Table S2) failed to grow on synthetic medium supplemented with ethanol and glycerol (SMEG, Fig. 5D, E, F, G, respectively). The inability of SjURA9-expressing strains to grow on SMEG resembled that of the respiratory-deficient strain IMK242 [52]. In contrast, strain IME603, which expressed ScURA1 from a multicopy plasmid, as well as ura1A strains expressing other URA9-expressing did grow on SMEG, (Additional file 5: Figure S3). Removal of the SjURA9 expression plasmid from strain IME571, yielding strain IMS1206, did not restore growth on these non-fermentable carbon sources (Fig. 5H).
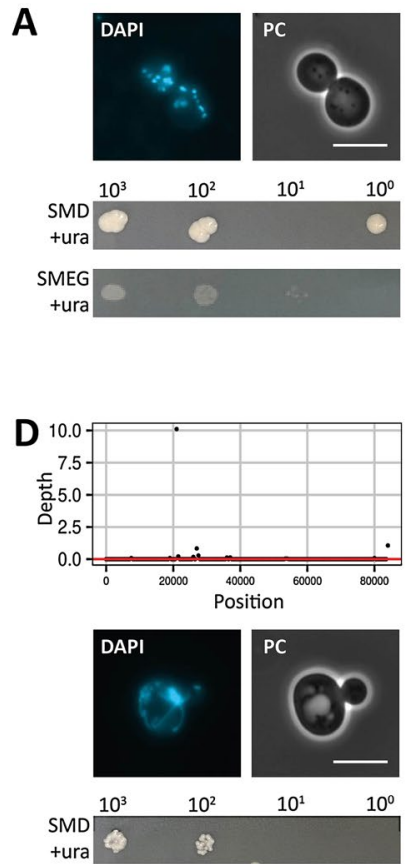

SMEG

+ura

\section{G}
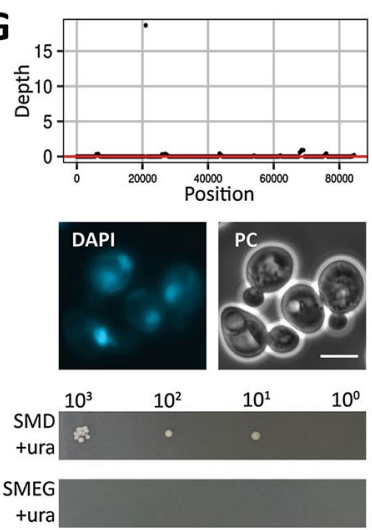

B
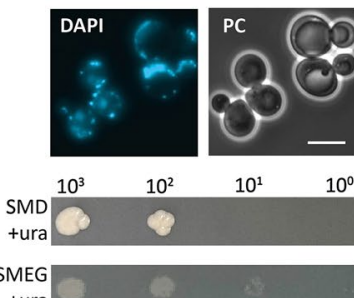

+ura
tuEG
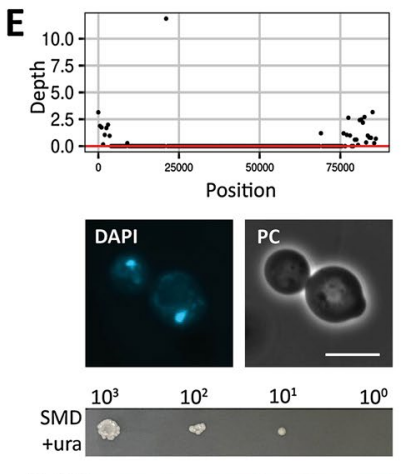

SMEG
+ura
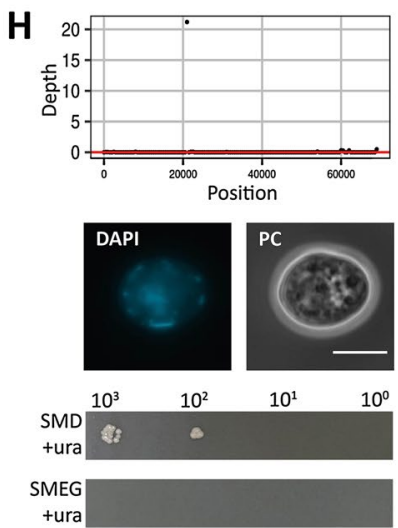

C
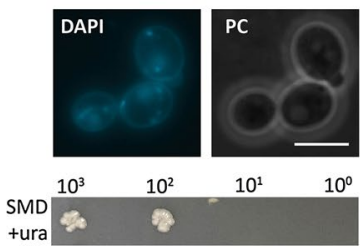

SMEG

F
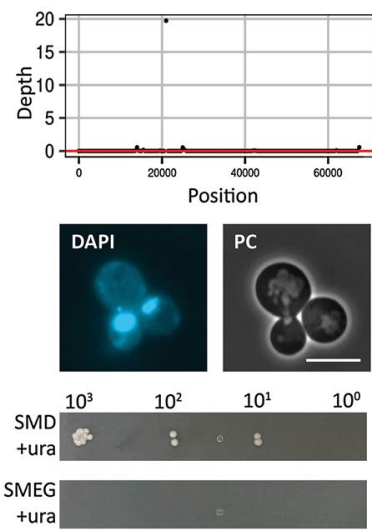

Fig. 5 Mitochondrial DNA integrity and respiratory capacity of strains expressing SjURA9. Top: sequencing coverage depth (average of non-overlapping $500 \mathrm{bp}$ sliding window of the mitochondrial genome) obtained by short-read sequencing of mitochondrial DNA relative to the reference strain CEN.PK113-7D. Middle: phase contrast (PC) microscopy and fluorescence of nuclei and mitochondrial nucleoids of yeast cells stained with the DNA-specific fluorescent dye DAPI; scale bars represent $5 \mu \mathrm{m}$. Bottom: spot plate assays on glucose-containing synthetic medium with uracil (SMUD + ura) and ethanol-glycerol containing synthetic medium with uracil (SMEG + ura). SMUD + ura and SMEG + ura plates were incubated at $30^{\circ} \mathrm{C}$ for 3 and 10 days respectively. Panels show data for the following yeast strains: A S. cerevisiae CENPK.113-7D (reference), B S. cerevisiae IME603 (multicopy ScURA 1), C S. cerevisiae IMK242 (rip1A::kanMX), D S. cerevisiae IMI452 (ura14::SjURA9, from IMX585), E S. cerevisiae IMI462

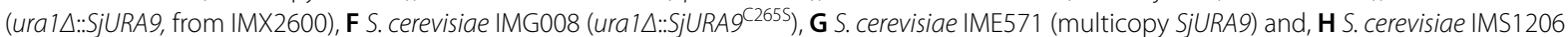
(IME571 cured from its SjURA9 expression plasmid) 
To explore whether the loss of respiratory competence in SjURA9-expressing strains was caused by loss of mitochondrial DNA, its presence was evaluated by staining with the DNA-specific dye 4',6-diamidino-2-phenylindole (DAPI, [53]) and by whole-genome sequencing. The reference S. cerevisiae strain CEN.PK113-7D (Fig. 5A) and a strain expressing ScURA1 from a multicopy plasmid (IME603; Fig. 5B) showed punctuated extranuclear DAPI staining. The SjURA9-expressing S. cerevisiae strains IMI462, IMG008 and IME571 only showed fluorescent staining of nuclear DNA, while strain IMI452 (ura1A::SjURA9) showed vague DAPI-stained tubular structures. Whole-genome sequencing showed absence of mitochondrial DNA in all the four strains SjURA9expressing S. cerevisiae strains (Fig. 5). These observations indicated that expression of SjURA9 in aerobic $S$. cerevisiae cultures causes loss of mitochondrial DNA and respiratory capacity.

\section{ArUra9 uses free FAD and FMN as electron acceptors}

The absence of mitochondria in Neocallimastigomycetes and the cytosolic localization of ArUra9-eGFP in S. cerevisiae implied that its activity was unlikely to involve components of the mitochondrial respiratory chain. To identify possible natural electron acceptors of ArUra9, enzyme-activity assays were performed in cell extracts of strain IME569 (ura1D mcArura9). Cell extracts of strains IME603 (mcScURA1) and IMK824 (ura1A) were included as references.

Consistent with literature reports [54], cell extracts that only contained the Class-I DHOD ScUra1 (strain IME603) showed dihydroorotate oxidation with fumarate as electron acceptor $\left(0.11 \mu \mathrm{mol} \cdot \mathrm{mg}\right.$ protein $\left.{ }^{-1} \cdot \mathrm{min}^{-1}\right)$, while a similar activity was observed with the artificial electron acceptor phenazine methosulfate (PMS, Table 3). ScUra1-containing cell extracts also showed dihydroorotate oxidation without addition of electron acceptor (Table 3), which was attributed to the previously reported ability of DHODs to use molecular oxygen as electron acceptor [55-58].

A high rate of PMS-dependent dihydroorotate oxidation $\left(0.13 \mu \mathrm{mol} \cdot \mathrm{mg}\right.$ protein $\left.{ }^{-1} \cdot \mathrm{min}^{-1}\right)$ confirmed DHODase activity in cell extracts of the ArUra9-expressing strain IME569. A low activity in the absence of an added electron acceptor (Table 3) suggested that, like other DHODs, ArUra9 can use molecular oxygen as electron acceptor. Assays in which fumarate, $\mathrm{NAD}^{+}, \mathrm{NADP}^{+}$or decylubiquinone were added to reaction mixtures did not increase activities beyond this basal level. Other compounds with a standard redox potential above or close to that of DHOD-bound FMN cofactors ( -242 to - $310 \mathrm{mV}$; [58-60]) were therefore tested. Addition of flavin adenine mononucleotide $\left(\mathrm{FAD} ; \mathrm{E}^{0}=-219 \mathrm{mV}\right.$
Table 3 Dihydroorotate-dehydrogenase activities in cell extracts of $\mathrm{S}$. cerevisiae strains expressing different DHODs, measured with different electron acceptors

\begin{tabular}{|c|c|c|c|}
\hline \multirow[t]{2}{*}{ Electron acceptor } & \multicolumn{3}{|c|}{ DHOD activity $\left(\mu \mathrm{mol} \cdot \mathrm{mg}\right.$ protein $\left.{ }^{-1} \cdot \mathrm{min}^{-1}\right)$} \\
\hline & $\begin{array}{l}\text { S. cerevisiae } \\
\text { IMK824 } \\
\text { ura14 }\end{array}$ & $\begin{array}{l}\text { S. cerevisiae } \\
\text { IME603 } \\
\text { mCSCURA1 }\end{array}$ & $\begin{array}{l}\text { S. cerevisiae } \\
\text { IME569 } \\
\text { ura } 1 \Delta \text { mcArura9 }\end{array}$ \\
\hline- & $<0.005$ & $0.010 \pm 0.001$ & $0.008 \pm 0.001$ \\
\hline PMS & $<0.005$ & $0.111 \pm 0.007$ & $0.132 \pm 0.029$ \\
\hline Fumarate & $<0.005$ & $0.110 \pm 0.020$ & $0.006 \pm 0.000$ \\
\hline $\mathrm{NAD}^{+}$ & $<0.005$ & $<0.005$ & $<0.005$ \\
\hline $\mathrm{NADP}^{+}$ & $<0.005$ & $<0.005$ & $<0.005$ \\
\hline$Q_{D}$ & $<0.005$ & $0.033 \pm 0.022$ & $0.009 \pm 0.003$ \\
\hline FMN & $<0.005$ & $0.037 \pm 0.002$ & $0.126 \pm 0.012$ \\
\hline FAD & $<0.005$ & $0.011 \pm 0.002$ & $0.148 \pm 0.032$ \\
\hline
\end{tabular}

Activities were measured in S. cerevisiae strains IMK824 (ura1D), IME603 (expressing ScURA1 from a multicopy plasmid) and in the strain IME569 (expressing Arura9 from a multicopy plasmid). Activities were measured without addition of electron acceptor (-), $0.1 \mathrm{mM}$ phenazine methosulfate (PMS), $1 \mathrm{mM}$ fumarate, $1 \mathrm{mM}$ nicotinamine adenine dinucleotide $\left(\mathrm{NAD}^{+}\right), 1 \mathrm{mM}$ nicotinamide adenine dinucleotide phosphate $\left(\mathrm{NADP}^{+}\right), 0.1 \mathrm{mM}$ decylubiquinone $\left(\mathrm{Q}_{\mathrm{D}}\right)$, $20 \mu \mathrm{M}$ flavin mononucleotide (FMN) or $20 \mu \mathrm{M}$ flavin adenine dinucleotide (FAD). Activities are represented as the average \pm mean deviation of activities measured with two independently prepared cell extracts

[61]), flavin mononucleotide $\left(\mathrm{FMN} ; \mathrm{E}^{\prime 0}=-219 \mathrm{mV}\right.$ [61]) strongly promoted dihydroorotate oxidation by cell extracts of the ArUra9-expressing strain IME569, with DHOD activities of $0.15 \mu \mathrm{mol} \cdot \mathrm{mg}$ protein ${ }^{-1} \cdot \mathrm{min}^{-1}$ and $0.13 \mu \mathrm{mol} \cdot \mathrm{mg}$ protein ${ }^{-1} \cdot \mathrm{min}^{-1}$, respectively. Supplementation of dihydroxyacetone phosphate (DHAP), acetaldehyde, pyruvate or oxaloacetate as electron acceptor did not increase enzyme activity (Additional file 5: Table S4).

\section{Discussion}

Fumarate-dependent Class-IA DHODs in S. cerevisiae (ScUra1) and closely related yeasts circumvent the oxygen requirement of respiration-dependent pyrimidine synthesis via the mitochondrial Class-II DHODs that occur in most other fungi $[19,20,22]$. The assumption that presence of a Class-IA DHOD is required for anaerobic pyrimidine prototrophy in fungi was first called into question when analysis of the genome of $D$. bruxellensis indicated absence of a Class-IA DHOD gene and, instead, revealed a sequence (DbURA9) with similarity to Class-II DHOD genes [17, 23, 28]. We identified similar situations in obligately anaerobic Neocallimastigomycetes and in the facultatively anaerobic fission yeast Sch. japonicus (Table 1, Fig. 1). Heterologous expression studies in ura1 $S$. cerevisiae showed that DbUra9, as well as orthologs from the Neocallimastigomycete A. robustus (ArUra9) and Sch. japonicus (SjUra9), supported anaerobic pyrimidine prototrophy. 
This phenotype was not observed for ura1 $S$. cerevisiae strains expressing URA9 genes from the aerobic yeasts K. marxianus and O. parapolymorpha (Fig. 2) nor in similar experiments involving URA9 genes from other aerobic yeasts $[8,19,25]$.

A cysteine as catalytic base is considered a hallmark of Class-I DHODs [57] while, with few reported exceptions, Class-II enzymes have a serine in this position [16]. Instead, the three anaerobically active Ura9 orthologs investigated in this study had a Cys in the catalyticbase position and shared this feature with only 3 of 331 other predicted fungal Ura9 orthologs. The latter three sequences originated from C. reversa, Sm. culicis and G. prolifera (Fig. 1), which were all originally isolated from microaerobic or anoxic environments (dung [62], insect guts [63] and submerged fruits [64], respectively). A Cysto-Ser change in the active site of Sch. japonicus SjUra9 specifically abolished its ability to support anaerobic pyrimidine prototrophy of S. cerevisiae (Fig. 3). While this result suggested that a Cys as catalytic base can be relevant for anaerobic functionality, changes at the same position of A. robustus ArUra9 (Cys to Ser) and K. marxianus KmUra9 (Ser to Cys) showed it is neither absolutely required nor sufficient for anaerobic activity of Class-II DHODs (Fig. 3).

Enzyme assays in cell extracts showed that ArAro9 can use free FAD and FMN as electron acceptors (Table 3), which was not previously observed for DHODs. Biochemical standard redox potentials of the non-enzyme-bound $\mathrm{FADH}_{2} / \mathrm{FAD}$ and $\mathrm{FMNH}_{2} /$ FMN redox couples $\left(E^{0,}=-219 \mathrm{mV}\right.$; [65]) and those of DHOD-bound $\mathrm{FMNH}_{2} / \mathrm{FMN}$ ( -242 to $-330 \mathrm{mV}$; [5860]) are compatible with either of these flavin cofactors acting as physiological electron acceptor of ArUra9. In anaerobic chemostat cultures of $S$. cerevisiae, intracellular FAD and FMN contents of 0.17 and $0.09 \mu \mathrm{mol}(\mathrm{g}$ biomass $)^{-1}$ were reported [66]. While use of free flavins in cellular redox reactions is relatively rare, the $S$. cerevisiae fumarate-reductase Osm1 can re-oxidize free $\mathrm{FADH}_{2}$ [67]. Since combined deletion of OSM1 and its paralog FRD1 abolishes anaerobic growth of S. cerevisiae [68], we could not experimentally verify their involvement in the in vivo anaerobic activity of ArUra9. Sequences with strong homology to S. cerevisiae Frd1 and/or Osm1 were found in Neocallimastigomycetes, Sch japonicus and D. bruxellensis (Additional file 5: Table S3). In silico prediction of subcellular localization indicated that these putative fumarate reductases were mitochondrial in Sch. japonicus and D. bruxellensis. Consistent with the inferred cytosolic localization of ArUra9, they were predicted to occur in the cytosol of Neocallimastigomycetes (Additional file 5: Table S3).
Previous studies implicated the $\mathrm{N}$-terminal domains of Class II DHODs in quinone binding [46, 69]. Based on analysis of 1500 Class II DHOD sequences and structural alignments, Sousa et al. [16] proposed conserved residues involved in quinone binding, stabilizing and pocket entry (Additional file 5: Figure S2). Ura9 sequences from Neocallimastigomycetes lacked several of these conserved residues as a consequence of their $\mathrm{N}$-terminal truncation. In addition, a conserved positively-charged residue implicated in quinone binding by other Class-II DHODs was replaced by a hydrophobic residue in ArUra9 and orthologs from other Neocallimastigomycetes (Additional file 5: Figure S2). These observations, together with the absence of quinone-dependent DHOD activity in cell extracts containing ArUra9 (Table 3) support the notion that, even though quinones have been detected in these anaerobes [70], they are not involved in the activity of ArUra9 and orthologs in other Neocallimastigomycetes,.

Consistent with the $\mathrm{N}$-terminal truncation of Ura9 orthologs from Neocallimastigomycetes (Additional file 5: Figure S2), an ArUra9-eGFP fusion expressed in S. cerevisiae was localized to the cytosol (Fig. 4). Sch. japonicus and D. bruxellensis Ura9 orthologs retained an $\mathrm{N}$-terminal mitochondrial targeting sequence, as well as conserved residues proposed to be involved quinone binding (Additional file 5: Figure S2). The only difference in quinone-associated residues was a Tyr-to-Phe change in SjUra9 (Y137), which, in view of the similarity of these amino acids, may not have affected functionality (Additional file 5: Figure S2). We were unable to measure activities of DbUra9 and ScUra9 in cell extracts with the artificial electron acceptor PMS or other potential electron acceptors.

DbUra9-expressing ura1s isolates of S. cerevisiae evolved for fast anaerobic pyrimidine-prototrophy revealed three different mutations in the FUM1 fumarase gene. Mutations in the human fumarase gene $(F H)$ have been implicated with different types of cancer due to increased fumarate concentrations [71]. Thr218 and Ala294 of DbUra9 correspond to Val197 and Ala274, respectively, in $\mathrm{FH}$ and are both located in highly conserved regions [72, 73], while Ala274 resides in the active site [74]. Mutation of Ala274 to a valine in human fumarate hydratase $(\mathrm{FH})$ was implicated in ovarian mucinous cystadenoma [75], and resulted in a 50\% decreased activity of the enzyme [73]. By analogy, it seems probable that the Fum $1^{\mathrm{A} 294 \mathrm{~V}}$ change in strain IMS1169 also led to a reduced fumarase activity. Corresponding amino acids in human FH of Thr218 and Met432 are located in the core helix, and accumulated fumarate resulting from mutations in this helix have been associated with different types of cancer [74]. If further research confirms higher intracellular 
fumarate concentrations in strains carrying FUM1 mutations, these might favor in vivo use of fumarate as electron acceptor, as in Class I-A enzymes [57] or, alternatively, stimulate reoxidation of $\mathrm{FADH}_{2}$ via fumarate reductases.

Anaerobic pyrimidine synthesis, combined with acquisition by horizontal gene transfer of genes enabling sterol-independent anaerobic growth (squalene hopene cyclase; [31]) and anaerobic deoxynucleotide synthesis (Class-I ribonucleotide reductase; [41]), indicates that Sch. japonicus is remarkably well adapted to anaerobic growth. The observation that independently constructed SjUra9-expressing S. cerevisiae strains consistently showed loss of respiratory capacity and mitochondrial DNA (Fig. 5) indicated a trade-off between anaerobic pyrimidine synthesis and respiratory competence. Quinone-dependent DHODs are known to react with oxygen, leading to formation of hydrogen peroxide and superoxide [55]. In S. cerevisiae, these reactive oxygen species have been implicated in loss of respiratory capacity [76]. Despite presence of respiratory proteins, including low levels of all cytochromes [76], Sch. japonicus strains are naturally respiratory deficient [77-79]. It is tempting to speculate whether this phenotype may have provided a driving force for evolution of a respirationindependent DHOD or, alternatively, be a consequence of evolution of SjUra9 for anaerobic functionality. With respect to the latter option, it should be considered that the heterologous context in which loss of respiratory competence was observed in this study does not necessarily reflect conditions in Sch. japonicus or its ancestors.

\section{Conclusions}

Our results show that, in addition to the well-established acquisition of a Class-I DHOD by S. cerevisiae and closely related yeasts, at least three separate events in fungal evolution enabled anaerobic pyrimidine synthesis by variants of Class-II DHODs that do not depend on aerobic respiration. These anaerobically active variants were shown to have a cysteine instead of a conserved serine residue in their catalytic sites. Their in vivo activities were not dependent on aerobic respiration, and, in Neocallimastigomycetes, they were not membrane associated and could use free FAD or FMN as electron acceptor. These remarkable differences with canonical Class-II DHODs underline the plasticity of fungal genomes and genes under selective pressure and extend our knowledge on eukaryotic adaptation to anoxic environments.

\section{Methods}

\section{Yeast strains, media and strain maintenance}

Saccharomyces cerevisiae strains were derived from the CEN.PK lineage (Additional file 5: Table S2; [80, 81]). O. parapolymorpha CBS11895, K. marxianus CBS6556 and
Sch. japonicus CBS5679 were obtained from the Westerdijk Institute (Utrecht, The Netherlands). Synthetic media with ammonium as nitrogen source (SM) and with urea as nitrogen source (SMU), containing vitamins and trace elements, were prepared and sterilized as described previously [82, 83]. A separately autoclaved (30 min, $\left.110{ }^{\circ} \mathrm{C}\right) \mathrm{D}$-glucose solution $(50 \% \mathrm{w} / \mathrm{v})$ was added to sterile SMU or SM at a concentration of $20 \mathrm{~g} \mathrm{~L}^{-1}$, yielding SMUD and SMD, respectively. SM with ethanol and glycerol (SMEG) and complex yeast extract-peptoneglucose medium (YPD) were prepared as described previously [84]. Where indicated, YPD was supplemented with $200 \mathrm{mg} \mathrm{L}^{-1}$ filter-sterilized geneticin (G418; Thermo Fisher Scientific, Waltham, MA) or hygromycin B (HygB; Thermo Fisher Scientific). Synthetic media for anaerobic growth experiments were supplemented with Tween 80 and ergosterol [82]. Uracil-auxotrophic strains were routinely grown on SMUD supplemented with $150 \mathrm{mg}$ $\mathrm{L}^{-1}$ uracil (SMD + ura) or, to obtain uracil-limited precultures, with $15 \mathrm{mg} \mathrm{L}^{-1}$ uracil (SMUD+ura0.1). Solid synthetic and complex media were prepared by adding $20 \mathrm{~g} \mathrm{~L}^{-1}$ Bacto agar (Difco laboratories Inc, Detroit, MI) prior to autoclaving. Escherichia coli strains were grown on Lysogeny Broth (LB; [85]), supplemented with $100 \mathrm{mg} \mathrm{L}^{-1}$ filter-sterilized ampicillin (Sigma Aldrich, St. Louis, MO) or chloramphenicol (Sigma Aldrich) as indicated (LB-amp and LB-cam, respectively). Frozen stock cultures of yeast strains were prepared as described previously [86] after growth to mid-exponential phase at $30{ }^{\circ} \mathrm{C}$ on YPD (strains CENPK.113-5D, CEN.PK1137D, IMX585, IMX2600, CBS6556 and CBS11895), on SMUD + ura (strain IMK824) or on SMUD (other strains). E. coli strains were grown at $37^{\circ} \mathrm{C}$ on LB-amp or LB-cam and frozen stock cultures were prepared as described by Mans et al. [86].

\section{Molecular biology techniques}

Phusion High Fidelity DNA Polymerase (Thermo Fisher Scientific) and PAGE-purified oligonucleotide primers (Additional file 5: Table S5, Sigma Aldrich) were used in polymerase chain reactions (PCRs) for cloning and sequencing. Diagnostic PCRs were performed with desalted oligonucleotides (Additional file 5: Table S5, Sigma Aldrich) and DreamTag Mastermix 2X (Thermo Fisher Scientific). Genomic DNA as template for PCRs was isolated using a YeaStar Genomic DNA kit (Zymo Research, Irvine, CA). PCR products were purified with a GeneElute PCR Clean-Up kit (Sigma Aldrich) or from $1 \%$ agarose gels using a Zymoclean Gel DNA Recovery Kit (Zymo Research). Gibson Assembly of purified DNA fragments with 20 bp sequence overlaps was performed with the NEBuilder HiFi DNA Assembly Mastermix (New England Biolabs, Ipswich, MA). Golden-Gate 
assembly was performed according to Lee et al. [87]. E. coli XL1-Blue (Agilent Technologies, Santa Clara, CA) was chemically transformed following manufacturer's instructions and plated on selective media. Correct plasmid assembly was verified by diagnostic PCRs on E. coli transformants [86]. Cas9-mediated genome editing in S. cerevisiae was performed according to Mans et al. [86]. The LiAc/SS-DNA/PEG method [88] was used for yeast transformation with plasmids (at least $1 \mu \mathrm{g}$ DNA per transformation) or linear DNA fragments $(0.5-1 \mu \mathrm{g}$ per transformation). Single-colony isolates of randomly picked transformants were obtained by three re-streaks on selective media. Integrations and deletions were verified by diagnostic PCRs with genomic DNA as a template. Construction of plasmids and yeast strains is described in detail in Additional file 6.

\section{Whole-genome DNA sequencing}

Genomic DNA of yeast strains was isolated from overnight cultures on YPD, except for strain IME571 that was grown on SMUD, using the Qiagen Genomic DNA 100/G kit (Qiagen, Hilden, Germany) with the Proteinase-K step extended to $3 \mathrm{~h}$. DNA concentrations were measured on a Qubit Fluorometer (Invitrogen, Carlsbad, CA, USA) with the QuBit BR dsDNA Assay kit (Invitrogen). Whole-genome sequencing on an Illumina MiSeq platform (Illumina Novoseq 6000, Illumina Inc., San Diego, CA, USA) was performed by Novogene Europe (Cambridge, UK; strains IMI439, IMS1167, IMS1168, IMS1169 and IMS1170), Macrogen Europe BV (Amsterdam, The Netherlands; strains IMG008, IME571 and IMS1206) or in-house (strains IMI452 and IMI462). For in-house sequencing, the Nextera DNA Flex 509 Library Prep kit (Illumina) was used for paired-end library preparation. Genome sequences were deposited at GenBank (BioProject accession number PRJNA745202).

\section{Whole-genome sequence analysis}

Sequencing data were processed as described previously [89]. For sequence analysis of the evolved strains IMS1167-IMS1170, their parental strain IMI439 was used as reference to obtain sequence differences. For IMI452/IMI462/IMG008 or IME571/IMS1206 IMI452 and IME571 were used as reference, respectively. Identified SNP's were individually checked with the Integrated Genomics Viewer (IGV; [90]). Mitochondrial DNA coverage plots were generated by calculating the average of non-overlapping 500 bp sliding windows ( $\mathrm{R}$ version 3.6.0).

\section{Protein sequence homology search and phylogenetic analysis}

Proteomes of $A$. robustus (NCBI taxid 1754,192), P. finnis (taxid 1754191), Neocallimastix californiae (1754190),
Sch. japonicus (402676), D. bruxellensis (5007; [91]), K. marxianus (1003335) and O. parapolymorpha (871575) were subjected to protein blast search in NCBI [92], using LkUra1 (UniProt KB accession number Q7Z892) and LkUra9 (Q6V3W9) as queries and applying default settings. Similarly, the proteomes of A. robustus, P. finnis, $N$. californiae, Sch. japonicus and that of D. bruxellensis were subjected to a protein blast using ScFrd1 (GenBank accession number EIW10990.1) or Osm1 (EIW09573.1) as query, default settings were applied.

To predict the localization of putative fumarate reductases in A. robustus, $N$. californiae, P. finnis, D. bruxellensis and Sch. japonicus, protein sequences were compared to known fungal sorting signals and motifs using the online computational tool WoLF PSORT [93]. The highest scoring cellular component for fungal settings was retrieved.

Bacterial and fungal amino acid sequences available from UniProt reference proteomes release 2019_02 [94] were systematically searched for Class-II dihydroorotate dehydrogenase orthologs. The database of fungal reference proteomes (NCBI taxid 4751) was supplemented with sequences available from the UniProt trembl division for the following organisms: D. bruxellensis (5007), Komagataella phaffii (981350), Komagataella pseudopastoris (169507), Komagataella pastoris (4922), Ogataea polymorpha (460523), Pichia membranifaciens (763406), Pichia kudriavzevii (4909), Neocallimastix californiae (1754190), P. finnis (1754191), A._robustus (1754192) and Piromyces sp. E2 (73868). Then, a ClassII DHOD of L. kluyveri CBS3082 (LkUra9; UniProt KB accession number Q6V3W9)[19] was used as query for a HMMER search [39], using cutoff values of 1e-5 and requiring hits to correspond to at least $75 \%$ of the query sequence length resulting in 724 fungal and 1595 bacterial Ura9 homologs. The sets of Ura9 homologs were further used to select a set of orthologs. For this purpose, the database of fungal proteomes was used to calculate all possible co-ortholog sets with proteinortho v6.0.25 [95] running diamond v2.0.8 [96], obtaining 331 Ura9 orthologs (Additional file 1). Similarly, the search for bacterial Ura9 orthologs resulted in 73 sequences (Additional file 1). Ura9-orthologous amino-acid sequences were then subjected to multiple sequence alignment using MAFFT v7.40286 [97] in "einsi" mode. Alignments were trimmed using trimAl v1.287 [98] in "gappyout" mode, and used to build a phylogenetic tree with RAxML-NG v0.8.188 [99] using 10 random and 10 parsimony starting trees, 100 Felsenstein Bootstrap replicates, and LG model. The resulting phylogenetic tree was visualized using iTOL (Interactive Tree Of Life) tool v6 [100]. 
Multiple sequence alignment of selected Ura9orthologs was performed in Clustal Omega [101] with default settings. Protein sequences of Ura9 orthologs were retrieved from the Uniprot database for Sch. pombe (SpUra3; Uniprot KB accession number P32747); L. kluyveri (LkUra9; Q6V3W9), O. parapolymorpha (OpUra9; W1QJ07), K. marxianus (KmUra9; Q6SZS6), E. coli (EcUra9; P0A7E1), D. bruxellensis (DbUra9; I2JUI3), Sch. japonicus (SjUra9; B6JXQ5), A. robustus (ArUra9; A0A1Y1XN91), N. californiae (NcUra9; A0A1Y2ELQ6) and $P$. finnis (PfUra9; A0A1Y1VDI5) and C. reversa (CmUra9; A0A2G5BHD4), Sm. culicis (ScuUra9; A0A1R1YI62) and G. prolifera (GpUra9; A0A139AY32).

\section{Cultivation of yeast strains}

Aerobic shake-flask cultures were grown in an Innova Incubator (New Brunswick Scientific, Edison, NJ) at $30{ }^{\circ} \mathrm{C}$ and $200 \mathrm{rpm}$. Pre-cultures in $100-\mathrm{mL}$ shake flasks with a working volume of $40 \mathrm{~mL}$ were inoculated with frozen stock cultures. Primary pre-cultures of yeast strains expressing plasmid-borne DHOD genes were grown on SMUD and those of other yeast strains on SMUD + ura, and were used to inoculate a secondary pre-culture on SMUD (for plasmid expressing strains) or SMUD + ura0.1. Upon reaching late exponential phase, cultures were centrifuged $(5 \mathrm{~min}$ at $3000 \times g)$ and washed twice with demineralized water. Washed cell suspensions were used to inoculate $500-\mathrm{mL}$ shake flasks containing $100 \mathrm{~mL}$ of SMUD + ura or SMUD, at an initial optical density at $660 \mathrm{~nm}\left(\mathrm{OD}_{660}\right)$ of 0.2 .

Anaerobic cultures were grown in $100-\mathrm{mL}$ shake flasks with a working volume of $80 \mathrm{~mL}$. Pre-cultures were grown aerobically on SMUD + ura as described above, until stationary phase, washed twice with sterile demineralized water and transferred to an anaerobic preculture on SMUD + ura0.1, supplemented with Tween 80 and ergosterol. Flasks were incubated on an IKA KS 260 orbital shaker (240 rpm; Dijkstra Verenigde BV, Lelystad, The Netherlands) placed in a Shel Lab Bactron 300 anaerobic workstation (Sheldon Manufacturing Inc, Cornelius, OR) at $30{ }^{\circ} \mathrm{C}$. The gas mixture supplied to the anaerobic workstation contained $10 \% \mathrm{CO}_{2}, 5 \% \mathrm{H}_{2}$ and $85 \% \mathrm{~N}_{2}$. Measures to minimize inadvertent oxygen entry were implemented as described by [102]. When anaerobic precultures reached stationary phase, they were used to inoculate cultures on SMUD and SMUD + ura supplemented with Tween 80 and ergosterol.

For spot-plate experiments, yeast strains were pregrown on $20 \mathrm{~mL}$ SMUD in $100-\mathrm{mL}$ shake flasks, centrifuged ( $5 \mathrm{~min}, 3000 \mathrm{~g}$ ) and washed twice with demineralized water. Washed cultures were used for cell counts with a Z2 Coulter particle count and size analyzer (Beckman Coulter, Brea, CA) set at particle size 2.5-7.5 $\mu \mathrm{m}$. Cells were diluted to a final concentration of $2.5 \cdot 10^{5}$ cells $\mathrm{mL}^{-1}$, and subsequently diluted to $2.5 \cdot 10^{4}$ cells $\mathrm{mL}^{-1}$, $2.5 \cdot 10^{3}$ cells $/ \mathrm{mL}$ and $2.5 \cdot 10^{2}$ cells $\mathrm{mL}^{-1}$. From these four dilutions, $4 \mu \mathrm{L}$ of each strain and dilution was transferred to SMD, SMD + ura, SMEG and SMEG + ura plates. All strains were pre-grown and plated in duplicate.

\section{Analytical methods}

Extracellular metabolite concentrations were measured by high performance liquid chromatography as described by Verhoeven et al. [103] Optical density at $660 \mathrm{~nm}$ of aerobic cultures was measured using an Jenway 7200 spectrophotometer (Bibby Scientific, Staffordshire, UK) after accurate dilution to an $\mathrm{OD}_{660}$ between 0.1 and 0.3 . Anaerobic cultures were first diluted to an optical density at $600 \mathrm{~nm}\left(\mathrm{OD}_{600}\right)$ between 0.15 and 0.35 , followed by optical densities measurements at $600 \mathrm{~nm}$ on an Ultrospec 10 cell density meter (Biochrom, Harvard Biosience, Holliston, MA) that was placed in the anaerobic workstation [102].

\section{Microscopy analysis and staining}

MitoTracker Deep Red FM (Invitrogen) staining was performed on early exponential phase aerobic cultures by adding $250 \mathrm{nM}$ MitoTracker Deep Red FM to a 1-mL culture sample and subsequent incubation in the dark at $37{ }^{\circ} \mathrm{C}$ for $15 \mathrm{~min}$. DNA staining was performed on $1-\mathrm{mL}$ samples of early exponential phase, aerobic cultures on $10 \mathrm{~mL}$ SMUD in 50-mL vented Greiner tubes (Greiner Bio-One, Kremsmünster, Austria). Cultures were supplemented with $300 \mathrm{nM} \mathrm{4',6-diamidino-2-phenylindole}$ (DAPI) dihydrochloride (Sigma Aldrich) and incubated in the dark for $10-15 \mathrm{~min}$ at $20^{\circ} \mathrm{C}$. Phase-contrast microscopy was performed using a Zeiss Axio Imager Z1 (Carl Zeiss AG, Oberkochen, Germany) that was equipped with a HAL 100 Halogen illuminator, HBO 100 illuminating system and AxioCam HRm Rev3 detector (60 N-C 1" $1.0 \times)$ (Carl Zeiss AG). The lateral magnification objective $100 \times / 1.3$ oil was used with Immersol $518 \mathrm{~F}$ type $\mathrm{F}$ immersion oil (Carls Zeiss AG). Fluoresence of eGFP was detected using filter set 10 (Carl Zeiss AG; excitation bandpass (BP) 470/20, emission 540/25). MitoTracker Deep Red and mRuby2 were imaged with filterset 14 (excitation BP 535/25, emission longpass (LP) 590) and 50 (excitation BP 640/30, emission BP 690/50) respectively (Carls Zeiss AG). For analysis of DAPI dihydrochloride fluorescence, filterset 49 (excitation Short Pass (SP) 380, emission BP 445/50) was used (Carl Zeiss AG). Results were analysed using the Fiji package of ImageJ [104]. 


\section{Preparation of cell extracts}

Strains carrying multi-copy plasmids expressing dihydroorotate dehydrogenases, were grown to mid-exponential phase in 100-mL shake flask cultures on SMUD. After centrifugation at $3000 \times g$ and at $0{ }^{\circ} \mathrm{C}$, biomass was resuspended in $4 \mathrm{~mL}$ ice-cold $10 \mathrm{mM}$ potassium phosphate buffer ( $\mathrm{pH} 7.5$ ) with $2 \mathrm{mM}$ EDTA and stored at $-20{ }^{\circ} \mathrm{C}$. Samples were thawed on ice, centrifuged at $3000 \times g$ and at $4{ }^{\circ} \mathrm{C}$, washed with $10 \mathrm{~mL}$ ice-cold sonication buffer (100 mM potassium phosphate buffer, $\mathrm{pH} 7.5$ with $2 \mathrm{mM}$ $\mathrm{MgCl}_{2}$ ) and resuspended in $4 \mathrm{~mL}$ sonication buffer containing 1 tablet of cOmplete Mini protease inhibitor (Sigma Aldrich) per $10 \mathrm{~mL}$ buffer. Cell extracts were prepared by sonication and centrifugation as described previously [105]. Bovine serum albumin (Sigma Aldrich) was used as a reference for analyses of protein concentrations in cell extracts [106].

\section{Dihydroorotate dehydrogenase activity assays in cell extracts}

Dihydroorotate dehydrogenase assays were performed at $30{ }^{\circ} \mathrm{C}$ in potassium phosphate buffer, $(100 \mathrm{mM}, \mathrm{pH} 7.5)$ using a Hitachi U-3010 UV/Visible spectrophotometer (Chiyoda, Tokyo, Japan). Formation of orotate or reduction of $\mathrm{NAD}(\mathrm{P})^{+}$was monitored by measuring absorbance at $300 \mathrm{~nm}\left(\varepsilon=3.05 \mathrm{mM}^{-1} \mathrm{~cm}^{-1}\right.$; [14]) or $340 \mathrm{~nm}$ $\left(\varepsilon=6.22 \mathrm{mM}^{-1} \mathrm{~cm}^{-1}\right.$; [107]) respectively, upon addition of $1 \mathrm{mM}$ dihydroorotate to a temperature-equilibrated reaction mixture containing buffer, cell extract and/or either of the electron acceptors fumarate (1 mM), decylubiquinone $\left(\mathrm{Q}_{\mathrm{D}}, 0.1 \mathrm{mM}\right.$, dissolved in dimethylsulfoxide), nicotinamide adenine dinucleotide $\left(\mathrm{NAD}^{+}, 1 \mathrm{mM}\right)$, nicotinamine adenine dinucleotide phosphate $\left(\mathrm{NADP}^{+}\right.$, $1 \mathrm{mM}$ ), flavine adenine dinucleotide (FAD, $20 \mu \mathrm{M}$ ), flavin mononucleotide (FMN, $20 \mu \mathrm{M})$ or the artificial electron acceptor phenazine methosulfate (PMS, $0.1 \mathrm{mM}$ ). Enzyme assays were performed on two separately prepared cell extracts. Reduction potentials of tested electron acceptors mentioned in the text are relative to the standard hydrogen electrode.

\footnotetext{
Abbreviations

Ar: Anaeromyces robustus; Db: Dekkera bruxellensis; DHAP: Dihydroxyacetone phosphate; DHOD: Dihydroorotate dehydrogenase; Cr: Coemansia reversa; Cys: Cysteine; FAD: Flavin adenine dinucleotide; FH: Fumarate hydratase; FMN: Flavin mononucleotide; GFP: Green fluorescent protein; Gp: Gonapodya prolifera; HGT: Horizontal gene transfer; Km: Kluyveromyces marxianus; Nc: Neocallimastix californiae; Op: Ogataea parapolymorpha; Pf: Piromyces finnis; PMS: Phenazine methosulfate; $Q_{D}$ : Decylubiquinone; Sc: Saccharomyces cerevisiae; Scu: Smittium culicus; Ser: Serine; SMD: Synthetic dextrose medium; SMEG: Synthetic ethanol/glycerol medium; SMUD: Synthetic urea medium with dextrose; Sj: Schizosaccharomyces japonicus; $\mu$ : Maximum specific growth rate.
}

\section{Supplementary Information}

The online version contains supplementary material available at https://doi. org/10.1186/s40694-021-00117-4.

Additional file 1. Protein sequences Ura9 orthologs.

Additional file 2. Protein IDs Ura9 orthologs.

Additional file 3. Raw phylogenetic tree.

Additional file 4. Codon-optimized protein sequences.

Additional file 5. Supplementary tables and figures.

Additional file 6. Plasmid and strain construction.

\section{Acknowledgements}

We gratefully thank Göktuğ Aba for construction of pUDC286 and Siem Eerden for construction of pUDE672 and pUDE677, Robert Stella for pUD538 and Petrik Buitenhuis for construction of pUDE815. We acknowledge Jisk van der Meer for constructing pUDR386, pUDE765, pUDE809, IMK839, IMI446, IMI447 and IMI452 and Magdalena Kaminska for construction of IMX2165, IMX2203, IMX2209, IMG005 and IMG007. We thank Marc Strampraad for the help with the enzyme assays and Sanne Wiersma and Nicole Bennis for their help with the aerobic growth study. We also thank Sanne Wiersma for critically reading the manuscript.

\section{Authors' contributions}

JB and JTP wrote the initial version of the manuscript. All authors contributed to the experimental design. JB, CCK and MAHL performed microscopy analysis, enzyme assays were performed by JB and MAHL Sequence analysis was performed by MB and JB and phylogenetic analysis by RAOM. Strain construction was performed by JB and MW and all growth experiments were performed by JB. All authors critically read the manuscript, provided input and approved the final manuscript.

\section{Funding}

This work was funded by an Advanced Grant of the European Research Council to JTP (Grant 555 694633).

\section{Availability of data and materials}

Figure 1 was made available online (https://itol.embl.de/export/1931902531 45446711626368835)Whole-genome sequencing data from strains IMS1167, IMS1 168, IMS1169, IMS1170, IMI452, IMI462, IMG008, IME571 and IMS1206 was deposited at NCBI (BioProject accession number PRJNA745202).

\section{Declarations}

Ethics approval and consent to participate

Not applicable.

Consent for publication

Not applicable.

Competing interests

We declare no conflicts of interest.

\section{Author details}

${ }^{1}$ Department of Biotechnology, Delft University of Technology, van der Maasweg 9, 2629 HZ Delft, The Netherlands. ${ }^{2}$ Wageningen Plant Research, Wageningen University and Research, Droevendaalsesteeg 1, 6708 PB Wageningen, The Netherlands.

Received: 29 July 2021 Accepted: 2 October 2021

Published online: 16 October 2021 


\section{References}

1. Larsen JN, Jensen KF. Nucleotide sequence of the pyrD gene of Escherichia coli and characterization of the flavoprotein dihydroorotate dehydrogenase. Eur J Biochem. 1985;151:59-65.

2. Kerr CT, Miller RW. Dihydroorotate-ubiquinone reductase complex of Escherichia coli B. J Biol Chem. 1968;243:2963-8.

3. Rowland P, Björnberg O, Nielsen FS, Jensen KF, Larsen S. The crystal structure of Lactococcus lactis dihydroorotate dehydrogenase A complexed with the enzyme reaction product throws light on its enzymatic function. Protein Sci. 1998;7:1269-79.

4. Rowland P, Nørager S, Jensen KF, Larsen S. Structure of dihydroorotate dehydrogenase B: electron transfer between two flavin groups bridged by an iron-sulphur cluster. Structure. 2000;8:1227-38.

5. Rawls J, Knecht W, Diekert K, Lill R, Löffler M. Requirements for the mitochondrial import and localization of dihydroorotate dehydrogenase. Eur J Biochem. 2000;267:2079-87.

6. Rowland P, Nielsen FS, Jensen KF, Larsen S. The crystal structure of the flavin containing enzyme dihydroorotate dehydrogenase A from Lactococcus lactis. Structure. 1997;5:239-52.

7. Reis RAG, Calil FA, Feliciano PR, Pinheiro MP, Nonato MC. The dihydroorotate dehydrogenases: past and present. Arch Biochem Biophys. 2017;632:175-91.

8. Nagy M, Lacroute F, Thomas D. Divergent evolution of pyrimidine biosynthesis between anaerobic and aerobic yeasts. Proc Natl Acad Sci U S A. 1992;89:8966-70.

9. Björnberg O, Grüner AC, Roepstorff P, Jensen KF. The activity of Escherichia coli dihydroorotate dehydrogenase is dependent on a conserved loop identified by sequence homology, mutagenesis, and limited proteolysis. Biochemistry. 1999;38:2899-908.

10. Jordan DB, Bisaha JJ, Picollelli MA. Catalytic properties of dihydroorotate dehydrogenase from Saccharomyces cerevisiae: Studies on $\mathrm{pH}$, alternate substrates, and inhibitors. Arch Biochem Biophys. 2000;378:84-92.

11. Fagan RL, Nelson MN, Pagano PM, Palfey BA. Mechanism of flavin reduction in class 2 dihydroorotate dehydrogenases. Biochemistry. 2006:45:14926-32.

12. Fagan $\mathrm{RL}$, Jensen $\mathrm{KF}$, Björnberg O, Palfey BA. Mechanism of flavin reduction in the class 1 A dihydroorotate dehydrogenase from Lactococcus lactis. Biochemistry. 2007:46:4028-36.

13. Björnberg O, Jordan DB, Palfey BA, Jensen KF. Dihydrooxonate is a substrate of dihydroorotate dehydrogenase (DHOD) providing evidence for involvement of cysteine and serine residues in base catalysis. Arch Biochem Biophys. 2001;391:286-94.

14. Nørager S, Jensen KF, Björnberg O, Larsen S. E. coli dihydroorotate dehydrogenase reveals structural and functional distinctions between different classes of dihydroorotate dehydrogenases. Structure. 2002;10:1211-23.

15. Nara T, Hshimoto T, Aoki T. Evolutionary implications of the mosaic pyrimidine-biosynthetic pathway in eukaryotes. Gene. 2000;257:209-22

16. Sousa FM, Refojo PN, Pereira MM. Investigating the amino acid sequences of membrane bound dihydroorotate:quinone oxidoreductases (DHOQOs): Structural and functional implications. Biochim Biophys Acta Bioenerg. 2021;1862: 148321.

17. Piškur J, Ling Z, Marcet-Houben M, Ishchuk OP, Aerts A, LaButti K, Copeland A, Lindquist E, Barry K, Compagno C, Bisson L, Grigoriev IV, Gabaldón T, Phister T. The genome of wine yeast Dekkera bruxellensis provides a tool to explore its food-related properties. Int J Food Microbiol. 2012;157:202-9.

18. Armenta-Medina D, Segovia L, Perez-Rueda E. Comparative genomics of nucleotide metabolism: a tour to the past of the three cellular domains of life. BMC Genomics. 2014;15:1-16.

19. Gojković Z, Knecht W, Zameitat E, Warneboldt J, Coutelis JB, Pynyaha Y, Neuveglise C, Møller K, Löffler M, Piškur J. Horizontal gene transfer promoted evolution of the ability to propagate under anaerobic conditions in yeasts. Mol Genet Genomics. 2004;271:387-93.

20. Hall C, Brachat S, Dietrich FS. Contribution of horizontal gene transfer to the evolution of Saccharomyces cerevisiae. Eukaryot Cell. 2005:4:1102-15.

21. Visser W, Scheffers WA, Batenburg-Van der Vegte WH, Van Dijken JP. Oxygen requirements of yeasts. Appl Environ Microbiol. 1990;56:3785-92.
22. Hagman A, Sall T, Compagno C, Piskur J. Yeast, "make-accumulateconsume" life strategy evolved as a multi-step process that predates the whole genome duplication yeast. PLoS ONE. 2013. https://doi.org/10. 1371/journal.pone.0068734

23. Riley R, Haridas S, Wolfe KH, Lopes MR, Hittinger CT, Göker M, et al. Comparative genomics of biotechnologically important yeasts. Proc Natl Acad Sci U S A. 2016:113:9882-7.

24. Annoura T, Nara T, Makiuchi T, Hashimoto T, Aoki T. The origin of dihydroorotate dehydrogenase genes of kinetoplastids, with special reference to their biological significance and adaptation to anaerobic, parasitic conditions. J Mol Evol. 2005;60:113-27.

25. Shi NQ, Jeffries TW. Anaerobic growth and improved fermentation of Pichia stipitis bearing a URA1 gene from Saccharomyces cerevisiae. Appl Microbiol Biotechnol. 1998:50:339-45.

26. Blomqvist J, Eberhard T, Schnürer J, Passoth V. Fermentation characteristics of Dekkera bruxellensis strains. Appl Microbiol Biotechnol. 2010;87:1487-97.

27. Tiukova IA, Petterson ME, Tellgren-Roth C, Bunikis I, Eberhard T, Pettersson OV, Passoth V. Transcriptome of the alternative ethanol production strain Dekkera bruxellensis CBS 11270 in sugar limited, low oxygen cultivation. PLoS ONE. 2013;8:2-8.

28. Woolfit M, Rozpȩdowska E, Piškur J, Wolfe KH. Genome survey sequencing of the wine spoilage yeast Dekkera (Brettanomyces) bruxellensis. Eukaryot Cell. 2007;6:721-33.

29. Blomqvist J, Passoth V. Dekkera bruxellensis-spoilage yeast with biotechnological potential, and a model for yeast evolution, physiology and competitiveness. FEMS Yeast Res. 2015:15:1-9.

30. Bulder CJEA. Anaerobic growth, ergosterol content and sensitivity to a polyene antibiotic, of the yeast Schizosaccharomyces japonicus. Antonie Van Leeuwenhoek. 1971;37:353-8.

31. Bouwknegt J, Wiersma SJ, Ortiz-Merino RA, Doornenbal ESR, Buitenhuis P, Giera M, Müller C, Pronk JT. A squalene-hopene cyclase in Schizosaccharomyces japonicus represents a eukaryotic adaptation to sterol-limited anaerobic environments. Proc Natl Acad Sci. 2021;118: e2105225118.

32. Yarlett N, Orpin CG, Munn EA, Greenwood CA. Hydrogenosomes in the rumen fungus Neocallimastix patriciarum. Biochem J. 1986;236:729-39.

33. Akhmanova A, Voncken FGJ, Hosea KM, Harhangi H, Keltjens JT, Op Den Camp HJM, Vogels GD, Hackstein JHP. A hydrogenosome with pyruvate formate-lyase: Anaerobic chytrid fungi use an alternative route for pyruvate catabolism. Mol Microbiol. 1999;32:1103-14.

34. Dekker WJC, Ortiz-Merino RA, Kaljouw A, Battjes J, Wiering FW, Mooiman C, de la Torre P. Engineering the thermotolerant industrial yeast Kluyveromyces marxianus for anaerobic growth. Metab Eng. 2021;67:347-64.

35. Dekker WJC, Juergens H, Ortiz-Merino RA, Mooiman C, van den Berg R, Mans R, Pronk JT. Re-oxidation of cytosolic NADH is a major contributor to the high oxygen requirements of the thermotolerant yeast Ogataea parapolymorpha in oxygen-limited cultures. BioRXiv. 2021. https://doi. org/10.1101/2021.04.30.442227.

36. Fonseca GG, Heinzle E, Wittmann C, Gombert AK. The yeast Kluyveromyces marxianus and its biotechnological potential. Appl Microbiol Biotechnol. 2008:79:339-54

37. Zameitat E, Gojković Z, Knecht W, Piškur J, Löffler M. Biochemical characterization of recombinant dihydroorotate dehydrogenase from the opportunistic pathogenic yeast Candida albicans. FEBS J. 2006;273:3183-91.

38. Löffler M, Knecht W, Rawls J, Ullrich A, Dietz C. Drosophila melanogaster dihydroorotate dehydrogenase: the N-terminus is important for biological function in vivo but not for catalytic properties in vitro. Insect Biochem Mol Biol. 2002;32:1159-69.

39. Mistry J, Finn RD, Eddy SR, Bateman A, Punta M. Challenges in homology search: HMMER3 and convergent evolution of coiled-coil regions. Nucleic Acids Res. 2013. https://doi.org/10.1093/nar/gkt263.

40. UniProt Consortium. Universal Protein Resource. 2002. https://www. uniprot.org/. Accessed 28 Nov 2019.

41. Lundin D, Gribaldo S, Torrents E, Sjöberg BM, Poole AM. Ribonucleotide reduction - horizontal transfer of a required function spans all three domains. BMC Evol Biol. 2010. https://doi.org/10.1186/ 1471-2148-10-383. 
42. Murphy CL, Youssef NH, Hanafy RA, Couger MB, Stajich JE, Wang Y, Baker K, Dagar SS, Griffith GW, Farag IF, Callaghan TM, Elshahed MS. Horizontal gene transfer as an indispensable driver for evolution of Neocallimastigomycota into a distinct gut dwelling fungal lineage. Appl Environ Microbiol. 2019;85:e00988-e1019.

43. Takishita K, Chikaraishi Y, Leger MM, Kim E, Yabuki A, Ohkouchi N, Roger AJ. Lateral transfer of tetrahymanol-synthesizing genes has allowed multiple diverse eukaryote lineages to independently adapt to environments without oxygen. Biol Direct. 2012. https://doi.org/10.1186/ 1745-6150-7-5.

44. Pronk JT. Auxotrophic yeast strains in fundamental and applied research. Appl Environ Microbiol. 2002;68:2095-100.

45. Haitjema CH, Solomon KV, Henske JK, Theodorou MK, O'Malley MA. Anaerobic gut fungi: advances in isolation, culture, and cellulolytic enzyme discovery for biofuel production. Biotechnol Bioeng. 2014;111:1471-82.

46. Liu S, Neidhardt EA, Grossman TH, Ocain T, Clardy J. Structures of human dihydroorotate dehydrogenase in complex with antiproliferative agents. Structure. 2000;8:25-33.

47. Hansen M. Inhibitor binding in a class 2 dihydroorotate dehydrogenase causes variations in the membrane-associated $\mathrm{N}$-terminal domain. Protein Sci. 2004;13:1031-42.

48. Fagan RL, Palfey BA. Roles in binding and chemistry for conserved active site residues in the class 2 dihydroorotate dehydrogenase from Escherichia coli. Biochemistry. 2009;48:7169-78.

49. Vowinckel J, Hartl J, Butler R, Ralser M. MitoLoc: a method for the simultaneous quantification of mitochondrial network morphology and membrane potential in single cells. Mitochondrion. 2015;24:77-86.

50. Visser W, van Spronsen EA, Nanninga N, Pronk JT, Kuenen JG, van Dijken JP. Effects of growth conditions on mitochondrial morphology in Saccharomyces cerevisiae. Antonie Van Leeuwenhoek. 1995;67:243-53.

51. Kováč L, Varečka L.' Membrane potentials in respiring and respirationdeficient yeasts monitored by a fluorescent dye. Biochim Biophys Acta Bioenerg. 1981;637:209-16.

52. Perez-Samper G, Cerulus B, Jariani A, Vermeersch L, Simancas NB, Bisschops MMM, van den Brink J, Solis-Escalante D, Gallone B, De Maeyer D, van Bael E, Wenseleers T, Michiels J, Marchal K, Daran-Lapujade P, Verstrepen KJ. The crabtree effect shapes the Saccharomyces cerevisiae lag phase during the switch between different carbon sources. MBio. 2018:9:1-18.

53. Kapuscinski JDAPI. A DNA-specific fluorescent probe. Biotech Histochem. 1995;70:220-33.

54. Andersen PS, Jansen PJG, Hammer K. Two different dihydroorotate dehydrogenases in Lactococcus lactis. J Bacteriol. 1994;176:3975-82.

55. Hey-Mogensen M, Goncalves RLS, Orr AL, Brand MD. Production of superoxide/ $\mathrm{H} 2 \mathrm{O} 2$ by dihydroorotate dehydrogenase in rat skeletal muscle mitochondria. Free Radic Biol Med. 2014;72:149-55.

56. Arakaki TL, Buckner FS, Gillespie JR, Malmquist NA, Phillips MA, Kalyuzhniy O, Luft JR, Detitta GT, Verlinde CLMJ, Van VWC, Hol WGJ, Ethan A. Characterization of Trypanosoma brucei dihydroorotate dehydrogenase as a possible drug target; structural, kinetic and RNAi studies. Mol Microbiol. 2008;68:37-50.

57. Björnberg O, Rowland P, Larsen S, Jensen KF. Active site of dihydroorotate dehydrogenase A from Lactococcus lactis investigated by chemical modification and mutagenesis. Biochemistry. 1997;36:16197-205.

58. Zameitat E, Pierik AJ, Zocher K, Löffler M. Dihydroorotate dehydrogenase from Saccharomyces cerevisiae: spectroscopic investigations with the recombinant enzyme throw light on catalytic properties and metabolism of fumarate analogues. FEMS Yeast Res. 2007;7:897-904.

59. Mohsen AWA, Rigby SEJ, Jensen KF, Munro AW, Scrutton NS. Thermodynamic basis of electron transfer in dihydroorotate dehydrogenase B from Lactococcus lactis: analysis by potentiometry, EPR spectroscopy, and ENDOR spectroscopy. Biochemistry. 2004;43:6498-510.

60. Palfey BA, Björnberg O, Jensen KF. Insight into the chemistry of flavin reduction and oxidation in Escherichia coli dihydroorotate dehydrogenase obtained by rapid reaction studies. Biochemistry. 2001;40:4381-90.

61. Schafer FQ, Buettner GR. Redox state and redox environment in biology. In: Forman HJ, Fukuto J, Torres M, editors. Signal transduction by reactive oxygen and nitrogen species: pathways and chemical principles. Dordrecht: Springer, Netherlands; 2003. p. 1-14.
62. Tiegh S, le Monn G. Coemansia reversa. Ann Sci Nat Bot. 1873;17:392.

63. Lastra CCL. Primera cita de Smittium culisetae y S. culicis

(Trichomycetes:Harpellales) en larvas de mosquitos (Diptera:culicidae) de la republica Argentina. Bol Soc Argent Bot. 1997;33:43-6.

64. Fuller MS, Clay RP. Observations of Gonapodya in pure culture: growth, development and cell wall characterization. Mycologia. 1993;85:38-45.

65. Draper RD, Ingraham LL. A potentiometric study of the flavin semiquinone equilibrium. Arch Biochem Biophys. 1968;125:802-8.

66. Seifar RM, Ras C, Deshmukh AT, Bekers KM, Suarez-Mendez C, da Cruz ALB, van Gulik WM, Heijnen JJ. Quantitative analysis of intracellular coenzymes in Saccharomyces cerevisiae using ion pair reversed phase ultra high performance liquid chromatography tandem mass spectrometry. J Chromatogr A. 2013;1311:115-20.

67. Kim S, Kim CM, Son YJ, Choi JY, Siegenthaler RK, Lee Y, Jang TH, Song J, Kang H, Kaiser CA, Park HH. Molecular basis of maintaining an oxidizing environment under anaerobiosis by soluble fumarate reductase. Nat Commun. 2018;9:1-12.

68. Arikawa Y, Enomoto K, Muratsubaki H, Okazaki M. Soluble fumarate reductase isoenzymes from Saccharomyces cerevisiae are required for anaerobic growth. FEMS Microbiol Lett. 1998;165:111-6.

69. Ullrich A, Knecht W, Fries M, Löffler M. Recombinant expression of $\mathrm{N}$-terminal truncated mutants of the membrane bound mouse, rat and human flavoenzyme dihydroorotate dehydrogenase: a versatile tool to rate inhibitor effects? Eur J Biochem. 2001;268:1861-8.

70. Youssef NH, Couger MB, Struchtemeyer CG, Liggenstoffer AS, Prade RA, Najar FZ, Atiyeh HK, Wilkins MR, Elshahed MS. The genome of the anaerobic fungus Orpinomyces sp. strain C1A reveals the unique evolutionary history of a remarkable plant biomass degrader. Appl Environ Microbiol. 2013;79:4620-34.

71. Linehan WM, Rouault TA. Molecular pathways: Fumarate Hydratasedeficient kindey cancer-targeting the Warburg effect in cancer. Clin Cancer Res. 2013;19:3346-52.

72. Pithukpakorn M, Wei MH, Toure O, Steinbach PJ, Glenn GM, Zbar B, Linehan WM, Toro JR. Fumarate hydratase enzyme activity in lymphoblastoid cells and fibroblasts of individuals in families with hereditary leiomyomatosis and renal cell cancer. J Med Genet. 2006;43:755-62.

73. Marque M, Gardie B, Bressac De Paillerets B, Rustin P, Guillot B, Richard $\mathrm{S}$, Bessis D. Novel FH mutation in a patient with cutaneous leiomyomatosis associated with cutis verticis gyrata, eruptive collagenoma and Charcot-Marie-Tooth disease. Br J Dermatol. 2010;163:1337-9.

74. Picaud S, Kavanagh KL, Yue WW, Lee WH, Muller-Knapp S, Gileadi O, Sacchettini J, Oppermann U. Structural basis of fumarate hydratase deficiency. J Inherit Metab Dis. 2011;34:671-6.

75. Ylisaukko-oja SK, Cybulski C, Lehtonen R, Kiuru M, Matyjasik J, Szymañska A, Szymañska-Pasternak J, Dyrskjot L, Butzow R, Orntoft TF, Launonen V, Lubiñski J, Aaltonen LA. Germline fumarate hydratase mutations in patients with ovarian mucinous cystadenoma. Eur J Hum Genet. 2006;14:880-3.

76. Doudican NA, Song B, Shadel GS, Doetsch PW. Oxidative DNA damage causes mitochondrial genomic instability in Saccharomyces cerevisiae. Mol Cell Biol. 2005:25:5196-204.

77. Bulder CJEA, Weijers C. Absence of cyanide-insenstive respiration in Schizosaccharomyces japonicus. FEMS Microbiol Lett. 1982;15:145-7.

78. Bulder CJEA. On respiratory deficiency in yeasts. Delft: Delft University of Technology; 1963.

79. Kaino T, Tonoko K, Mochizuki S, Takashima Y, Kawamukai M. Schizosaccharomyces japonicus has low levels of $\mathrm{CoQ} 10$ synthesis, respiration deficiency, and efficient ethanol production. Biosci Biotechnol Biochem. 2018;82:1031-42.

80. Entian KD, Kötter P. 25 Yeast genetic strain and plasmid collections. Methods Microbiol. 2007;36:629-66.

81. Salazar AN, de Vries ARG, van den Broek M, Wijsman M, Cortés PdlT, Brickwedde A, Brouwers N, Daran JMG, Abeel T. Nanopore sequencing enables near-complete de novo assembly of Saccharomyces cerevisiae reference strain CEN.PK113-7D. FEMS Yeast Res. 2017;17:1-11.

82. Verduyn C, Postma E, Scheffers WA, van Dijken JP. Effect of benzoic acid on metabolic fluxes in yeasts: a continuous-culture study on the regulation of respiration and alcoholic fermentation. Yeast. 1992;8:501-17.

83. Luttik MAH, Kötter P, Salomons FA, van der Klei IJ, van Dijken JP, Pronk JT. The Saccharomyces cerevisiae ICL2 gene encodes a mitochondrial 
2-methylisocitrate lyase involved in propionyl-coenzyme a metabolism. J Bacteriol. 2000;182:7007-13.

84. Mans R, Hassing EJ, Wijsman M, Giezekamp A, Pronk JT, Daran JM, van Maris AJA. A CRISPR/Cas9-based exploration into the elusive mechanism for lactate export in Saccharomyces cerevisiae. FEMS Yeast Res. 2017;17:1-12.

85. Bertani G. Studies on lysogenesis. I. The mode of phage liberation by lysogenic Escherichia coli. J Bacteriol. 1951;62:293-300.

86. Mans R, van Rossum HM, Wijsman M, Backx A, Kuijpers NGA, van den Broek M, Daran-Lapujade P, Pronk JT, van Maris AJA, Daran JM-G. CRISPR/Cas9: a molecular Swiss army knife for simultaneous introduction of multiple genetic modifications in Saccharomyces cerevisiae. FEMS Yeast Res. 2015;15:fov004.

87. Lee ME, DeLoache WC, Cervantes B, Dueber JE. A highly characterized yeast toolkit for modular, multipart assembly. ACS Synth Biol. 2015:4:975-86.

88. Gietz RD, Woods RA. Transformation of yeast by lithium acetate/singlestranded carrier DNA/polyethylene glycol method. Methods Enzymol. 2002;350:87-96.

89. Perli T, Vos AM, Bouwknegt J, Dekker WJC, Wiersma SJ, Mooiman C, Ortiz-Merino RA, Daran J-M, Pronk JT. Identification of oxygen-independent pathways for pyridine nucleotide and Coenzyme A synthesis in anaerobic fungi by expression of candidate genes in yeast. MBio. 2021:12:e00967-e1021.

90. Robinson JT, Thorvaldsdóttir H, Winckler W, Guttman M, Lander ES, Getz G, Mesirov JP. Integrative genome viewer. Nat Biotechnol. 2011;29:24-6.

91. Roach MJ, Borneman AR. New genome assemblies reveal patterns of domestication and adaptation across Brettanomyces (Dekkera) species. BMC Genomics. 2020;21:1-14.

92. Altschul SF, Wootton JC, Gertz ME, Agarwala R, Morgulis A, Schäffer AA, Yu Y-K. Protein database searches using compositionally adjusted substitution matrices. FEBS J. 2005;270:5101-9.

93. Horton P, Park KJ, Obayashi T, Fujita N, Harada H, Adams-Collier CJ, Nakai K. WoLF PSORT: protein localization predictor. Nucleic Acids Res. 2007:35:585-7.

94. The UniProt Consortium. UniProt: a worldwide hub of protein knowledge. Nucleic Acids Res. 2019;47:D506-15.

95. Lechner M, Findeiß S, Steiner L, Marz M, Stadler PF, Prohaska SJ. Proteinortho: detection of ( $\mathrm{CO}$ - ) orthologs in large-scale analysis. BMC Bioinform. 2011;12:1-9.

96. Buchfink B, Reuter K, Drost HG. Sensitive protein alignments at tree-oflife scale using DIAMOND. Nat Methods. 2021:18:366-8.

97. Katoh K, Standley DM. MAFFT multiple sequence alignment software version 7: improvements in performance and usability. Mol Biol Evol. 2013;30:772-80

98. Capella-Gutiérrez S, Silla-Martínez JM, Gabaldón T. trimAl: a tool for automated alignment trimming in large-scale phylogenetic analyses. Bioinformatics. 2009;25:1972-3.

99. Kozlov AM, Darriba D, Flouri T, Morel B, Stamatakis A. RAxML-NG: a fast, scalable and user-friendly tool for maximum likelihood phylogenetic inference. Bioinformatics. 2019:35:4453-5.

100. Letunic I, Bork P. Interactive tree of life (iTOL) v3: an online tool for the display and annotation of phylogenetic and other trees. Nucleic Acids Res. 2016;44:W242-5.

101. Sievers F, Wilm A, Dineen D, Gibson TJ, Karplus K, Li W, Lopez R, McWilliam H, Remmert M, Söding J, Thompson JD, Higgins DG. Fast, scalable generation of high-quality protein multiple sequence alignments using Clustal Omega. Mol Syst Biol. 2011;7:539.
102. Mooiman C, Bouwknegt J, Dekker WJC, Wiersma SJ, Ortiz-Merino RA, de Hulster EAF, Pronk JT. Critical parameters and procedures for anaerobic cultivation of yeasts in bioreactors and anaerobic chambers. FEMS Yeast Res. 2021;21(5):foab035

103. Verhoeven MD, Lee M, Kamoen L, van den Broek M, Janssen DB, Daran J-MG, van Maris AJA, Pronk JT. Mutations in PMR1 stimulate xylose isomerase activity and anaerobic growth on xylose of engineered Saccharomyces cerevisiae by influencing manganese homeostasis. Sci Rep. 2017:7:46155.

104. Schindelin J, Arganda-Carreras I, Frise E, Kaynig V, Longair M, Pietzsch T, Preibisch S, Rueden C, Saalfeld S, Schmid B, Tinevez J-Y, White DJ, Hartenstein V, Eliceiri K, Tomancak P, Cardona A. Fiji-an open platform for biological image analysis. Nat Methods. 2012;9:676-82.

105. Vuralhan Z, Luttik MAH, Tai SL, Boer VM, Morais MA, Schipper D, Almering MJH, Kötter P, Dickinson JR, Daran JM, Pronk JT. Physiological characterization of the ARO10-dependent, broad-substrate-specificity 2-oxo acid decarboxylase activity of Saccharomyces cerevisiae. Appl Environ Microbiol. 2005;71:3276-84.

106. Lowry OH, Rosebrough NJ, Farr AL, Randall RJ. Protein determination with the folin phenol reagent. J Biol Chem. 1951:193:265-76.

107. Bernofsky C, Wanda SY. Formation of reduced nicotinamide adenine dinucleotide peroxide. J Biol Chem. 1982;257:6809-17.

108. Haitjema CH, Gilmore SP, Henske JK, Solomon KV, de Groot R, Kuo $A$, et al. A parts list for fungal cellulosomes revealed by comparative genomics. Nat Microbiol. 2017;2:17087.

109. Mondo SJ, Dannebaum RO, Kuo RC, Louie KB, Bewick AJ, LaButti K, et al. Widespread adenine N6-methylation of active genes in fungi. Nat Genet. 2017:49:964-8.

110. Rhind N, Chen Z, Yassour M, Thompson DA, Haas BJ, Habib N, et al. Comparative functional genomics of the fission yeasts. Science. 2011:332(6032):930-6.

111. Dujon B, Alexandraki D, André B, Ansorge W, Baladron V, Ballesta JPG, et al. Complete DNA sequence of yeast chromosome XI. Nature. 1994;369:371-8.

112. Lertwattanasakul N, Kosaka T, Hosoyama A, Suzuki Y, Rodrussamee N, Matsutani M, Murata M, Fujimoto N, Suprayogi, Tsuchikane K, Limtong S, Fujita N, Yamada M. Genetic basis of the highly efficient yeast Kluyveromyces marxianus: Complete genome sequence and transcriptome analyses. Biotechnol Biofuels. 2015;8:1-14.

113. Ravin NV, Eldarov MA, Kadnikov VV, Beletsky AV, Schneider J, Mardanova ES, Smekalova EM, Zvereva MI, Dontsova OA, Mardanov AV, Skryabin KG. Genome sequence and analysis of methylotrophic yeast Hansenula polymorpha DL1. BMC Genomics. 2013;14:837.

\section{Publisher's Note}

Springer Nature remains neutral with regard to jurisdictional claims in published maps and institutional affiliations.

Ready to submit your research? Choose BMC and benefit from

- fast, convenient online submission

- thorough peer review by experienced researchers in your field

- rapid publication on acceptance

- support for research data, including large and complex data types

- gold Open Access which fosters wider collaboration and increased citations

- maximum visibility for your research: over 100M website views per year

At BMC, research is always in progress.

Learn more biomedcentral.com/submissions 